\title{
A Generalization of Eichler Integrals and Certain Local Systems over Spin Riemann Surfaces
}

\author{
In the memory of Professor Michio Kuga
}

By

Kyoji SAITo*

\begin{abstract}
Let $\left(X, K_{X}^{1 / 2}\right)$ be a spin Riemann surface*) of genus $\geq 2$. By using infinite dimensional representations of the fundamental group of $X$, we obtain many local systems on $X$, which taken together define a resolution of the halfcanonical ring of $X$ and indicate a non-abelian theory of abelian integrals on $X$. The work has a root in a study of the complex structure on the Fricke moduli space [14].

*) a Riemann surface together with a halfcanonical bundle (4.1).
\end{abstract}

\section{Summary of Results}

Let $\left(X, K_{X}^{1 / 2}\right)$ be a spin Riemann surface of genus $\geq 2$. For each $n \geq 0$, we construct an increasing sequence $F_{X, i}^{n} i \in \mathbb{Z}_{\geq 0}$ of finite dimensional C-vector subsheaves contained in the $\mathcal{O}_{X}\left(K_{X}^{-n / 2}\right)$, regarded as local systems over $X$ (cf. (4.3) and (5.3)). They are characterized by the following properties.

a) The initial local system $F_{X, 0}^{n}$ for $i=0$ is isomorphic to the one induced from $n$th symmetric tensor product $\operatorname{Sym}^{n}\left(\mathbf{C}^{2}\right)$ of the vector representation space $\mathbf{C}^{2}$ of $S L(2, \mathbf{R})$ (i.e. $\left.F_{X, 0}^{n} \simeq\left(\mathbf{H} \times \operatorname{Sym}^{n}\left(\mathbf{C}^{2}\right)\right) / \pi_{1}(X)\right)$.

b) Set $G_{X, i}^{n}:=F_{X, i+1}^{n} / F_{X, i}^{n}$ for $n, i \geq 0$. Then each graded piece $G_{X, i}^{n}$ is a trivial local system and the initial one $G_{X, 0}^{n}$ is isomorphic to $H^{0}\left(X, \mathcal{O}\left(K_{X}^{n / 2+1}\right)\right)$ for $i=0$ (5.4.5).

c) The cohomology of the $F_{X, i}^{n}$ for $n, i \geq 0$ is as follows ((2.2.5), (3.5.1) and (4.4) Lemma):

Received May 30, 1990.

1991 Mathematics Subject Classification: 14H30, 14H60, 30F10, 30F35.

* Research Institute for Mathematical Sciences, Kyoto University, Kyoto 606, Japan. 


$$
H^{*}\left(X, F_{X, i}^{n}\right) \simeq H^{*}\left(X, \mathcal{O}_{X}\left(K_{X}^{-n / 2}\right)\right) \oplus G_{X, i}^{n}[1] \oplus G_{X, i-1}^{n}[2] .
$$

d) The sum $\mathscr{G}_{X}:=\bigoplus_{i}^{\oplus} \oplus_{n} G_{X, i}^{n}$ forms a (infinitely generated) graded ring over (a part of) the halfcanonical ring $\mathscr{G}_{X, 0}:=\bigoplus_{n=0}^{\infty} G_{X, 0}^{n}$. Each graded piece $\mathscr{G}_{X, i}$ $=\bigoplus_{n} G_{X, i}^{n}$ for $i \geq 0$ is a finite $\mathscr{G}_{X, 0}$-module $(\S 6(6.1)$ and (6.2)).

e) There are derivation maps $\delta^{0}: \mathscr{G}_{X} \rightarrow \mathscr{G}_{X} \otimes_{\mathrm{Z}} H^{1}(X, \mathbb{Z})$ and $\delta^{1}: \mathscr{G}_{X} \otimes_{\mathbb{Z}} H^{1}(X, \mathbb{Z}) \rightarrow\left(\mathscr{G}_{X} \oplus F_{X, 0}^{0}\right) \otimes_{\mathbb{Z}} H^{2}(X, \mathbb{Z})$ (3.6) such that the following sequence (6.3.1) gives a resolution of the halfcanonical ring $\mathscr{G}_{X, 0}$ :

$$
0 \longrightarrow \mathscr{G}_{X, 0} \longrightarrow \mathscr{G}_{X} \stackrel{\delta^{0}}{\longrightarrow} \mathscr{G}_{X} \otimes_{\mathbb{Z}} H^{1}(X) \stackrel{\delta^{1} \oplus^{2}}{\longrightarrow}\left(\mathscr{G}_{X} \oplus F_{X, 0}^{0}\right) \otimes_{\mathbf{Z}} H^{2}(X) \longrightarrow 0 .
$$

where the map $l$ to the factor $F_{X, 0}^{0} \simeq \mathbb{C}$ is given by abelian integrals (6.3.2):

$$
f \in \operatorname{Hom}_{\mathbb{Z}}\left(\Gamma_{g}, G_{X, 0}^{0}\right) \longmapsto \sum_{i=1}^{g}\left(\int_{b_{i}} f\left(a_{i}\right)-\int_{a_{i}} f\left(b_{i}\right)\right) \in \mathbb{C}
$$

f) Statement e) implies the following generating formula for the classes $\left[G_{X, i}^{n}\right]$ in the Grothendieck-group of mapping class group equivariant vector spaces ((3.7) Corollary and (4.4) Lemma).

$$
\begin{aligned}
& \sum_{i=0}^{\infty}\left[G_{X, i}^{0}\right] t^{i}=\frac{\left[H^{0}\left(X, \mathcal{O}_{X}\left(K_{X}\right)\right)\right]-\left[H^{2}\left(X, \mathbf{C}_{X}\right)\right] t}{1-\left[H^{1}\left(X, \mathbf{C}_{X}\right)\right] t+\left[H^{2}\left(X, \mathbf{C}_{X}\right)\right] t^{2}} \quad \text { for } n=0, \\
& \sum_{i=0}^{\infty}\left[G_{X, i}^{n}\right] t^{i}=\frac{\left[H^{0}\left(X, \mathcal{O}_{X}\left(K_{X}^{n / 2+1}\right)\right)\right]}{1-\left[H^{1}\left(X, \mathbf{C}_{X}\right)\right] t+\left[H^{2}\left(X, \mathbf{C}_{X}\right)\right] t^{2}} \quad \text { for } n>0 .
\end{aligned}
$$

\section{Contents}

$\S 1$ Introduction

$\S 2$ The minimal module $\mathscr{F}=\left(F_{i}\right)_{i \geq 0}$

$\S 3$ The case of surface group

$\S 4$ The Eichler-Shimura isomorphism

$\S 5$ The Local systems over a spin Riemann surface

$\S 6$ The Algebra structure on $\mathscr{H}_{X}$ Appendix

Notation. Let $A$ be an algebra with 1 and $\Gamma$ a group. An $A-\Gamma$ module is a module with commuting left $A$ and right $\Gamma$ actions. An $A-\Gamma$ homomorphism is a map that commutes with the $A-\Gamma$ actions. A $\Gamma$-module $M$ is reduced if $M^{\Gamma}$ $:=\{m \in M: m \cdot \gamma=m$ for any $\gamma \in \Gamma\}$ is equal to 0 . The $i$ th cohomology group of $\Gamma$ with coefficients in an $A-\Gamma$ module $M$ is denoted by $H^{i}(\Gamma, M)$, which is of course an $A$-module.

By $\mathbb{C}$, we mean the complex number field and $\mathbb{H}:=\{z \in \mathbb{C}: \operatorname{Im}(z)>0\}$ is the 
complex upper half plane, whose coordinate is denoted by $z$.

By $\mathcal{O}_{X}$ and $K_{X}$ we denote the sheaf of germs of holomorphic functions (resp. the canonical line bundle) of a complex manifold $X$.

\section{§1. Introduction}

(1.1) The Teichmüller space as a real manifold is well known to carry a complex structure (Weil, Ahlfors, Bers [2]). The complex structure can be recovered by several different approaches: Earle \& Eells, Beilinson, Manin \& Schechtman [1], Hitchin [8], Pekornen [13], Tromba [18], Wolpert [20]. Although the approaches are different, each construction uses some analysis, or to put it better, uses some infinite-dimensional spaces. In contrast, the symplectic structure on the Teichmüller space is algebraic (cf. [7]).

(1.2) We recently showed [14] that the complex structure on the Teichmüller space can be recovered in terms of representations of Fuchsian groups into an infinite-dimensional space. The starting point of the present paper is an attempt to "approximate" this infinite-dimensional space by finite-dimensional subspaces. We first explain this in (1.2).

Let $\Gamma_{g}$ be the surface group of genus $g \geq 2$ and $\rho: \Gamma_{g} \rightarrow S L(2, \mathbf{R})$ a faithful discrete representation. The $\rho$ determines a Riemann surface $\rho\left(\Gamma_{g}\right) \backslash \mathbf{H}$ and conjugacy class $[\rho]$ of $\rho$ determines a point in the moduli space of spin Riemann surfaces of genus $g$ (cf. $\S 4$ ). The tangent space of the moduli at $[\rho]$ is identified with the cohomology group $H^{1}\left(\Gamma_{g}, \mathfrak{g}\right)$ (Weil [19]), where $\Gamma_{g}$ acts on $\mathrm{g}:=\operatorname{sl}(2, \mathbf{R})$ by the adjoint action of $S L(2, \mathbf{R})$ composed with $\rho$. The space acquires a complex structure via the Eichler-Shimura isomorphism $([5,15])$ :

$$
l_{*}: H^{1}\left(\Gamma_{g}, \mathfrak{g}\right) \simeq H^{1}\left(\Gamma_{g}, \Gamma(\mathbf{H}, \Theta)\right) .
$$

Here $\Gamma(\mathbf{H}, \Theta)$ is the complex vector space of holomorphic vector fields on the upper half-plane $\mathbf{H}, \Gamma_{g}$ acts on $\Gamma(\mathbf{H}, \Theta)$ by pulling back vector fields and $l_{*}$ is induced by the infinitesimal action $\imath: \mathfrak{g} \rightarrow \Gamma(\mathbf{H}, \Theta), x \mapsto(-1, z) X\left(\begin{array}{l}z \\ 1\end{array}\right) \frac{d}{d z}$. The integrability of this almost complex structure is proved directly in [14] (cf. [10]).

The $\Gamma_{g}$-action on $\Gamma(\mathbf{H}, \Theta)$ is neither unitary nor completely reducible. Hence one is led to look for a "small" $\mathbf{C}-\Gamma_{g}$ module $\mathscr{F}$ with $\mathfrak{g} \otimes_{\mathbf{R}} \mathbf{C} \subset \mathscr{F}$ $\subset \Gamma(\mathbf{H}, \Theta)$ such that the inclusion maps induce isomorphisms: $H^{1}\left(\Gamma_{g}, \mathfrak{g}\right)$ $\simeq H^{1}\left(\Gamma_{g}, \mathscr{F}\right) \simeq H^{1}\left(\Gamma_{g}, \Gamma(\mathbf{H}, \Theta)\right)$. We will see that there exists a smallest such $\mathscr{F}_{\rho}$ depending on the representation $\rho$. Furthermore, there exists an increasing sequence $\left(F_{\rho, i}\right)_{i \geq 0}$ of $\mathbf{C}-\Gamma_{g}$ modules of finite rank such that $\mathscr{F}_{\rho}=\bigcup_{i=0}^{\infty} F_{\rho, i}$ and $F_{\rho, i+1} / F_{\rho, i}$ for $i \geq 0$ are trivial $\Gamma_{g}$ modules. We call $\mathscr{F}_{\rho}$ the minimal module attached to the Eichler-Shimura isomorphism (*). 
(1.3) The construction of (1.2) can be carried out in the same way for the representations of $\Gamma_{g}$ on the infinite-dimensional spaces $\Gamma\left(\mathbf{H}, \mathcal{O}\left(K^{-n / 2}\right)\right)$ for $n \geq 0$ with the role of $g$ replaced by $\operatorname{Sym}^{n}\left(\mathbf{R}^{2}\right)$, where $n=2$ is the case discussed in (1.2) (see §4). The present paper studies minimal modules $\mathscr{F}_{\rho}^{n}$ attached to this situation. The minimal module $\mathscr{F}_{\rho}^{n}$ is again exhausted by an increasing sequence $\left\{F_{\rho, i}^{n}\right\}_{i \geq 0}$ of $\mathbb{C}-\Gamma_{g}$ modules of finite rank such that $F_{\rho, i+1}^{n} / F_{\rho, i}^{n}$ for $i \geq 0$ are trivial $\Gamma_{g}$ modules. The first step $F_{\rho, 1}^{n}$ of the sequence is already known as the space of Eichler integrals and $F_{\rho, 1}^{n} / F_{\rho, 0}^{n} \simeq \Gamma\left(\mathbf{H}, \mathcal{O}\left(K^{n / 2+1}\right)\right)^{\Gamma_{g}}$ (cf. (4.4) Remark and (5.4.5)). Therefore we regard the spaces $F_{\rho, i}^{n}$ for $i>0$ as generalizations of this and called them higher Eichler integrals.

Geometrically, the representation $\rho: \Gamma_{g} \rightarrow S L(2, \mathbf{R})$ determines a Riemann surface $X:=\rho\left(\Gamma_{g}\right) \backslash H$, together with halfcanonical (spin) bundle $K_{X}^{1 / 2}$ (c.f. (4.1)). Then $F_{X, i}^{n}:=\left(\mathrm{H} \times F_{\rho, i}^{n}\right) / \Gamma_{g}$ for $n, i \geq 0$ are local systems on $X$ contained in $\mathcal{O}_{X}\left(K^{-n / 2}\right)$, which are shown to have the properties stated in the Summary.

Although each step of the construction and proofs is carried out in terms of elementary use of group cohomology, as a whole, it seems that we are treating some new object, which may be summarized in a word: non-abelian theory of abelian integrals on a spin Riemann surface. To describe elements of $F_{\rho, i}^{n}$ as global holomorphic forms on $\mathbf{H}$ (as solutions of algebraic differential equations) seems to be an interesting but hard problem, which we do not consider in this paper.

Many of the constructions in this paper may be carried out for further classes of discrete groups, including groups acting on higher dimensional spaces. Some Lemmas are formulated in this generality. It would be interesting to clarify the meaning of the generalization of the complex $\left(\mathscr{G}_{X} \otimes_{\mathbf{Z}} H^{*}(\Gamma), \delta^{*}\right)$ in the Summary e) for such a discrete group $\Gamma$.

(1.4) The construction of the paper is as follows.

$\S 2$ treats the construction of a minimal module in a general setting. $\S 3$ is devoted to a calculation of the cohomology of a surface group $\Gamma_{g}$ by introducing the concept of $i$-regularity. $\S 4$ recalls the Eichler-Shimura isomorphism and shows that the associated minimal modules $\mathscr{F}_{\rho}^{n}=\left(F_{\rho, 1}^{n}\right)_{i \geq 0}$ satisfy the regularity condition of $\S 3$. In $\S 5$, we show that the $F_{\rho, i}^{n}$ form local systems over the Riemann surface $\Gamma_{g} \backslash \mathbf{H}$, depending only on the spin of $\rho$. The $F_{\rho, i}^{n}$ are understood as solution spaces of some linear differential equations. We study the algebra structure on $\mathscr{H}_{\rho}:=\bigoplus_{n=0}^{\infty} \mathscr{F}_{\rho}^{n} / F_{\rho, 0}^{n}$ and on $\operatorname{gr}\left(\mathscr{H}_{\rho}\right) \simeq \bigoplus_{n=0}^{\infty} \operatorname{gr}\left(\mathscr{F}_{\rho}^{n}\right)$ in §6. The Appendix treats the $\mathbb{Z}$-structure on $G_{X, i}^{n}$ for $n>0, i \geq 0$.

(1.5) The author is grateful to Prof. Y. Ihara for pointing out that (1.2) (*) is known as the Eichler-Shimura isomorphism, which is the starting point of the present work. He thanks Prof. M. Kashiwara and Prof. T. Oda for discussions and for drawing attention to a Theorem of Labute $[22,1]$ and its 
generalization due to Kohno and Oda [23, Theorem (1.4)] (cf. Summary f) and (2.6)).

He also thanks Prof. M. Reid for carefully reading the manuscript and for revising the English.

\section{§2. The Minimal Module $\mathscr{F}=\left(F_{i}\right)_{i \geq 0}$}

Let $\Gamma$ be a finitely generated group and $R$ an $\mathbf{R}-\Gamma$ module of finite rank. In (2.1) we consider a setting, in which the cohomology group $H^{1}(\Gamma, R)$ acquires a C-structure. Then Lemma (2.2) associates with this situation a construction of a filtered $\mathrm{C}-\Gamma$ module $\mathscr{F}=\left(F_{i}\right)_{i \geq 0}$, which we shall call the minimal module.

(2.1) Definition. A pair $(\mathscr{S}, l)$ consisting of a $\mathbf{C}-\Gamma$ module $\mathscr{S}$ and an $\mathbf{R}-\Gamma$ homomorphism $l: R \rightarrow \mathscr{S}$ will be called a complexification of $R$, if $\imath$ induces

i) an injective $\mathbf{C}-\Gamma$ homomorphism $\mathbf{C} \otimes_{\mathbf{R}} R \rightarrow \mathscr{S}$,

ii) a $\mathbf{C}$-isomorphism

$\mathrm{C} \otimes_{\mathrm{R}} R^{\Gamma} \simeq \mathscr{S}^{\Gamma}$

iii) an $\mathbf{R}$-isomorphism

$l_{*}: H^{1}(\Gamma, R) \simeq H^{1}(\Gamma, \mathscr{S})$.

(2.2) Lemma. Let $R$ be an $\mathbf{R}-\Gamma$ module of finite rank and $(\mathscr{S}, \imath)$ a complexification of $R$.

1. There exists a $\mathrm{C}-\Gamma$ submodule $\mathscr{F}$ of $\mathscr{S}$ having an increasing filtration $\left(F_{i}\right)_{i=0}^{\infty}$ by C- $\Gamma$ modules of finite rank such that $\mathscr{F}=\bigcup_{i=0}^{\infty} F_{i}$ and

i) $l$ induces an isomorphism:

$$
\mathbf{C} \otimes_{\mathbf{R}} l: \mathbf{C} \otimes_{\mathbf{R}} R \simeq F_{0} .
$$

ii) For each $i \in \mathbf{Z}_{\geq 0}$, the module $F_{i+1}$ is the largest $\mathbf{C}-\Gamma$ subspace of $\mathscr{F}$ containing $F_{i}$ such that $F_{i+1} / F_{i}$ is a $\Gamma$-trivial module.

iii) The inclusion map $\imath: R \rightarrow \mathscr{F}$ induces an $\mathbf{R}$-isomorphism

$$
H^{1}(\Gamma, R) \simeq H^{1}(\Gamma, \mathscr{F})
$$

2. The pair $(\mathscr{F}, \imath)$ with the properties i)-iii) is unique and rigid in the following two senses.

i) Let $\mathscr{H}$ be any $\mathbf{C}-\Gamma$ submodule of $\mathscr{S}$ containing $R$ such that the natural maps $H^{1}(\Gamma, R) \rightarrow H^{1}(\Gamma, \mathscr{H}) \rightarrow H^{1}(\Gamma, \mathscr{S})$ are isomorphisms. Then $\mathscr{F} \subset \mathscr{H}$.

ii) If $\varphi: \mathscr{F} \rightarrow \mathscr{F}$ is a $\mathbf{C}-\Gamma$ homomorphism which commutes with $l$, then $\varphi$ preserves the filtration on $\mathscr{F}$ and $\operatorname{gr}(\varphi): \operatorname{gr}(\mathscr{F}) \rightarrow \operatorname{gr}(\mathscr{F})$ is the identity. If $\mathscr{S}$ is reduced, then $\varphi$ itself is the identity.

Proof 1. Define the sequence $F_{i}$ by induction on $i=0,1, \ldots$.

$$
\begin{aligned}
& F_{0}:=\mathbf{C} l(R)=\imath(R) \oplus \sqrt{-1} l(R) \\
& F_{i+1}:=\left\{f \in \mathscr{S}: f \cdot \gamma-f \in F_{i} \text { for all } \gamma \in \Gamma\right\} \text { for } i \geq 0 .
\end{aligned}
$$


By construction, the $F_{i}$ are $\mathbb{C}-\Gamma$ modules, and induced action of $\Gamma$ on $F_{i+1} / F_{i}$ is trivial. Thus the properties i) and ii) are shown. where

Recall that the first cohomology group $H^{1}(\Gamma, \mathscr{S})$ is given by $Z^{1}(\Gamma, \mathscr{S}) / \delta \mathscr{S}$,

$$
Z^{1}(\Gamma, \mathscr{S}):=\{c: \Gamma \rightarrow \mathscr{S} \mid c(\gamma \delta)=c(\gamma) \delta+c(\delta)\}
$$

and $\delta(f)(\gamma):=f \cdot \gamma-f$ (for $f \in \mathscr{S}$ and $\gamma \in \Gamma$ ). Hence $\operatorname{ker}(\delta)=\mathscr{S}^{\Gamma}$.

Using this notation, the definition of the $F_{i}$ can be rewritten as

$$
F_{i+1}:=\delta^{-1}\left(Z^{1}\left(\Gamma, F_{i}\right)\right) \quad i \geq 0 .
$$

Thus $\delta$ induces bijection: $F_{i+1} / \mathscr{S}^{\Gamma} \stackrel{\cong}{\rightarrow} Z^{1}\left(\Gamma, F_{i}\right) \cap \delta(\mathscr{S})$ for $i \geq 0$. If $F_{i}$ is of finite rank, then so is $Z^{1}\left(\Gamma, F_{i}\right)$ since $\Gamma$ is finitely generated, and hence so is also $F_{i+1} / \mathscr{S}^{\Gamma}$. Since $\mathscr{S}^{\Gamma} \subset R \otimes \mathbb{C}$ is of finite rank, one concludes $F_{i+1}$ is of finite rank.

The inclusions $R \subset F_{i} \subset \mathscr{S}$ induce maps $H^{1}(\Gamma, R) \rightarrow H^{1}\left(\Gamma, F_{i}\right) \rightarrow H^{1}(\Gamma, \mathscr{S})$, whose compose is an $\mathbb{R}$-isomorphism by assumption. This implies that $Z^{1}\left(\Gamma, F_{i}\right)$ maps surjectively to $H^{1}(\Gamma, \mathscr{S})$; the kernel is by definition $F_{i+1} / \mathscr{S}^{\Gamma}$. Hence one has an exact sequence:

$$
0 \longrightarrow F_{i+1} / \mathscr{S}^{\Gamma} \stackrel{\delta}{\longrightarrow} Z^{1}\left(\Gamma, F_{i}\right) \longrightarrow H^{1}(\Gamma, \mathscr{S}) \longrightarrow 0, \text { for } i \geq 0,
$$

and a splitting as $\mathbb{C}$-vector spaces:

$$
H^{1}\left(\Gamma, F_{i}\right) \simeq H^{1}(\Gamma, \mathscr{S}) \oplus F_{i+1} / F_{i}
$$

for $i \geq 1$, where the projection $H^{1}\left(\Gamma, F_{i}\right) \rightarrow H^{1}(\Gamma, \mathscr{S})$ is induced by $F_{i} \subset \mathscr{S}$ and the inclusion $H^{1}(\Gamma, \mathscr{S}) \subset H^{1}\left(\Gamma, F_{i}\right)$ by $F_{i-1} \rightarrow F_{i}$. (A decomposition of the form (2.2.5) for $i=0$ will be treated in (2.4).)

We define $\mathscr{F}$ as the union $\mathscr{F}:=\bigcup_{i=0}^{\infty} F_{i}$. The inductive limit of (2.2.5) gives an isomorphism $H^{1}(\Gamma, \mathscr{F}) \simeq H^{1}(\Gamma, \mathscr{S})$ and hence (2.2.2).

2. i) Let $\mathscr{H}$ be a $\mathbb{C}-\Gamma$ submodule of $\mathscr{S}$ containing $R$ such that the inclusion $\mathscr{H} \subset \mathscr{S}$ induces an isomorphism: $H^{1}(\Gamma, \mathscr{H}) \simeq H^{1}(\Gamma, \mathscr{S})$. We prove the inclusion $F_{i} \subset \mathscr{H}$ by induction. The case $i=0$ is the assumption. The inclusion maps $F_{i} \subset \mathscr{H} \subset \mathscr{S}$ induce maps: $H^{1}\left(\Gamma, F_{i}\right) \rightarrow H^{1}(\Gamma, \mathscr{H}) \simeq H^{1}(\Gamma, \mathscr{S})$. Recalling (2.2.4), one obtains that $\delta\left(F_{i+1}\right)=\delta(\mathscr{H}) \cap Z^{1}\left(\Gamma, F_{i}\right)$. This implies that $\delta\left(F_{i+1}\right) \subset \delta(\mathscr{H})$ and hence $F_{i+1} / \mathscr{S}^{\Gamma} \subset \mathscr{H} /\left(\mathscr{H} \cap \mathscr{S}^{\Gamma}\right)$. Since $\mathscr{S}^{\Gamma} \subset F_{0} \subset \mathscr{H}$, one has $F_{i+1} \subset \mathscr{H}$.

ii) Let $\varphi: \mathscr{F} \rightarrow \mathscr{F}$ be a $\mathbb{C}-\Gamma$ homomorphism commuting with $l$. We show by induction on $i$ that the restriction $\varphi \mid F_{i}$ (for $i \geq 0$ ) is of the form $i d+\phi_{i}$, where $\phi_{i}$ is a map from $F_{i}$ to $F_{i-1}$. When $i=0$, we have $\phi_{0}=0$ by the assumption on $\varphi$. For $i \geq 0$, apply the induction hypothesis to the element $f \cdot \gamma$ 
$-f \in F_{i}$ for $f \in F_{i+1}$ and $\gamma \in \Gamma$. For all $\gamma \in \Gamma$, one gets a relation $\varphi(f) \cdot \gamma-\varphi(f)$ $=\varphi(f \cdot \gamma-f)=f \cdot \gamma-f+\phi_{i}(f \cdot \gamma-f)$, which we rewrite as

$$
(f-\varphi(f))-((f-\varphi(f))) \cdot \gamma= \begin{cases}\phi_{i}(f \cdot \gamma-f) \in F_{i-1} & \text { if } i>0 \\ 0 & \text { if } i=0 .\end{cases}
$$

Thus $f-\varphi(f) \in F_{i}$ and hence $\left.\varphi\right|_{F_{i+1}} \equiv i d\left(\bmod F_{i}\right) . \quad$ If $\mathscr{S}$ is reduced, then $\phi_{i}=0$ by induction. This completes the proof.

Remark 1. The condition 1. ii) of the Lemma is necessary for the unicity of the filtration $\left(F_{i}\right)_{i \in \mathbf{N}}$. This is equivalent to the exactness of the sequence:

$$
0 \longrightarrow F_{i+1} / F_{i} \stackrel{\delta}{\longrightarrow} H^{1}\left(\Gamma, F_{i}\right) \longrightarrow H^{1}(\Gamma, \mathscr{F}) \longrightarrow 0 \text { for } i \geq 0 \text {. }
$$

2. The complex structure $J$ on $H^{1}(\Gamma, R)$ induced from $H^{1}(\Gamma, \mathscr{S})$ is not enough to determine the $\mathbf{C}-\Gamma$ module $\mathscr{F}=\left(F_{i}\right)_{i \geq 0}$, since the short exact sequence (2.2.4) depends not only on $J$ but on the data of the projection: $Z^{1}\left(\Gamma, F_{i}\right)$ $\rightarrow H^{1}(\Gamma, \mathscr{S})\left(\simeq H^{1}(\Gamma, R)\right)$

(2.3) Definition. The filtered $\mathbf{C}-\Gamma$ module $(\mathscr{F}, \imath)$ introduced in Lemma (2.2) is called the minimal module relative to $(\mathscr{S}, \imath)$. The induced complex structure on $H^{1}(\Gamma, R)$ will be denoted by $J$.

(2.4) The complex structure $J$ on $H^{1}(\Gamma, R)$ determines the first term $F_{1} / F_{0}$ as follows. Let the setting be as in Lemma (2.2).

Lemma bis. The image of $F_{1} / F_{0}$ in $\mathbf{C} \otimes_{\mathbf{R}} H^{1}(\Gamma, R)$ under the map $\delta$ (2.2.6) is the eigenspace of $J$ for the eigenvalue $-\sqrt{-1}$. Hence one has a direct sum decomposition:

$$
F_{1} / F_{0} \oplus \overline{F_{1} / F_{0}} \stackrel{\delta \oplus \bar{\delta}}{\simeq} \mathbf{C} \otimes_{\mathbf{R}} H^{1}(\Gamma, R)
$$

and an isomorphism:

$$
\overline{F_{1} / F_{0}} \simeq H^{1}(\Gamma, \mathscr{S}),
$$

where $\bar{A}$ denotes the complex conjugate of a $\mathrm{C}$-vector space $A$.

Proof. The definitions of $F_{0}:=\mathbb{C} \otimes_{\mathbf{R}} R$ and $F_{1}$ in (2.2) give rise to the following commutative diagram 


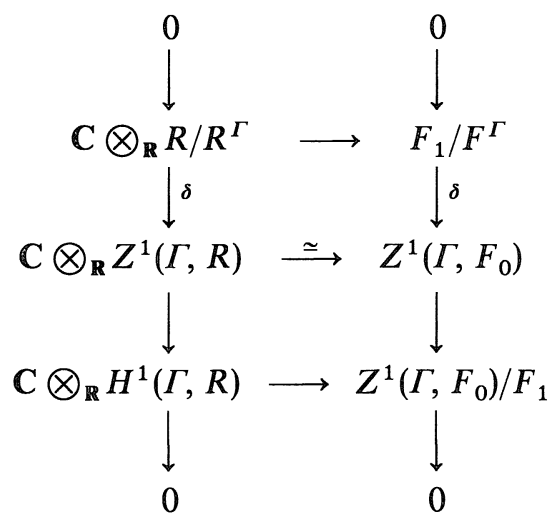

with exact columns. Then one has the exact sequence:

$$
0 \longrightarrow \operatorname{ker}\left(\mathbf{C} \otimes_{\mathbf{R}} H^{1}(\Gamma, R) \longrightarrow Z^{1}\left(\Gamma, F_{0}\right) / F_{1}\right) \rightarrow \operatorname{coker}\left(\mathbf{C} \otimes_{\mathbf{R}} R \rightarrow F_{1}\right) \longrightarrow 0 .
$$

The $Z^{1}\left(\Gamma, F_{0}\right) / F_{1}$ in the first parenthesis is isomorphic to $H^{1}(\Gamma, \mathscr{F})$ as a $\mathbb{C}$ vector space ((2.2.4)). The image of $\mathbb{C} \otimes_{\mathbb{R}} R$ in the second parenthesis is $F_{0} / F^{\Gamma}$ ((2.2.1)). These imply the isomorphism $F_{1} / F_{0} \simeq \operatorname{ker}\left(\mathbf{C} \otimes_{\mathbf{R}} H^{1}(\Gamma, R) \rightarrow H^{1}\right.$ $(\Gamma, \mathscr{F}))$. Hence one obtains the exact sequence:

$$
0 \longrightarrow F_{1} / F_{0} \longrightarrow H^{1}(\Gamma, R) \otimes \mathbb{C} \longrightarrow H^{1}(\Gamma, \mathscr{F}) \longrightarrow 0
$$

For an element $u:=x+\sqrt{-1} y \in H^{1}(\Gamma, R) \oplus \sqrt{-1} H^{1}(\Gamma, R)$, we have $l(u)$ $=0 \Longleftrightarrow l(x)+\sqrt{-1} l(y)=\imath(x+J y)=0 \Longleftrightarrow x+J y=0 \Longleftrightarrow u=x$ $+\sqrt{-1} J x\langle J u=-\sqrt{-1} u$.

(2.5) The coboundary map $\delta$ (cf. (3.6)).

Let the setting be as in (2.2) and let $\mathscr{F}=\left(F_{i}\right)_{i \geq 0}$ be a minimal module with its filtration. For $i \geq 0$ we define a map:

$$
F_{i+1} / F_{i} \stackrel{\delta}{\longrightarrow} F_{i} / F_{i-1} \otimes_{\mathbf{z}} H^{1}(\Gamma, \mathbb{Z}),
$$

called the coboundary map. In view of $\operatorname{Hom}(\Gamma, \mathbb{Z}) \cong H^{1}(\Gamma, \mathbb{Z})$, we identify the target space with $\operatorname{Hom}\left(\Gamma, F_{i} / F_{i-1}\right)$. Set

$$
\delta([f])(\gamma): \equiv f \cdot \gamma-f \quad \bmod \left(F_{i-1}\right)
$$

for $\gamma \in \Gamma$ and $f \in F_{i+1}$ representing $[f] \in F_{i+1} / F_{i}$. Here we write

$$
F_{-1}:=\sum_{\gamma \in \Gamma} F_{0} \cdot(\gamma-1)
$$

$\delta([f])$ is a homomorphism, since

$$
\delta([f])\left(\gamma_{1} \cdot \gamma_{2}\right) \equiv f \cdot \gamma_{1} \cdot \gamma_{2}-f
$$




$$
\begin{aligned}
& \equiv\left(f \cdot \gamma_{1}-f\right)+\left(f \cdot \gamma_{2}-f\right)+\left(\left(f \cdot \gamma_{1}-f\right) \cdot \gamma_{2}-\left(f \cdot \gamma_{1}-f\right)\right) \\
& \equiv \delta([f])\left(\gamma_{1}\right)+\delta([f])\left(\gamma_{2}\right) .
\end{aligned}
$$

The map $\delta(\text { for } \mathrm{i}>0)^{*)}$ is part of a long exact sequence:

$$
\begin{aligned}
0 \longrightarrow F_{i+1} / F_{i} \stackrel{\delta}{\longrightarrow} \operatorname{Hom}_{\mathbf{Z}}\left(\Gamma, F_{i} / F_{i-1}\right) & \longrightarrow H^{2}\left(F_{i-1}\right) \\
& \longrightarrow H^{2}\left(F_{i}\right) \longrightarrow H^{2}\left(F_{i} / F_{i-1}\right)
\end{aligned}
$$

Proof. For brevity, we drop $\Gamma$ in the notation for cohomology. Consider the long exact sequence associated to the short exact sequence $0 \rightarrow F_{i-1} \rightarrow F_{i}$ $\rightarrow F_{i} / F_{i-1} \rightarrow 0$ (for $i>0$ ):

$$
0 \longrightarrow F_{i} / F_{i-1} \rightarrow H^{1}\left(F_{i-1}\right) \longrightarrow H^{1}\left(F_{i}\right) \longrightarrow H^{1}\left(F_{i} / F_{i-1}\right) \longrightarrow H^{2}\left(F_{i-1}\right) \longrightarrow \cdots
$$

Since $F_{i} / F_{i-1}$ is $\Gamma$-trivial, one has $H^{1}\left(F_{i} / F_{i-1}\right)=\operatorname{Hom}_{\mathbf{z}}\left(\Gamma, F_{i} / F_{i-1}\right)$. Applying (2.2.6) and (2.2.5) for $H^{1}\left(F_{i}\right)$, one eliminates the first two terms, so that one obtains a sequence of the form (2.5.3) for some map $\delta$. Since the isomorphism of (2.2.5) on the second factor is induced by a coboundary map $\delta$, one gets the description of $\delta$ given in (2.5.1).

*) Note that (2.5.3) does not hold for $i=0$.

(2.6) Remark. Let $\mathbf{Z}[\Gamma]$ be the group algebra for a group $\Gamma$ and let $\mathscr{I}$ be its ideal generated by $\gamma-1$ for all $\gamma \in \Gamma$. Let $\mathscr{F}=\bigcup_{i=0}^{\infty} F_{i}$ be a minimal module (2.3). Then for any integers $i \geq 0$ and $j \geq 1$, the action of $\mathbf{Z}[\Gamma]$ on the quotient module $F_{i+j} / F_{i}$ is factored through an action of $\mathbf{Z}[\Gamma] / \mathscr{I}^{j}$. Therefore, the action of $\Gamma$ on $F_{i+j} / F_{i}$ is factored through $\Gamma / \Gamma_{j}$, where $\Gamma_{j}$ is the $j$ th lower central series of $\Gamma$. That is: $\Gamma_{1}:=\Gamma$ and $\Gamma_{j+1}:=\left[\Gamma, \Gamma_{j}\right]$ for $j \geq 1$.

Compare the formula f) in the Summary of the present paper with a Theorem of Labute $[22,1]$ and its generalization due to Kohno and Oda [23, Theorem (1.4)].

\section{§3. The Case of Surface Group}

We calculate the cohomology of the surface group of genus $g>1$

$$
\Gamma_{g}:=\left\langle a_{1}, b_{1}, \ldots, a_{g}, b_{g} \mid \prod_{j=1}^{g}\left[a_{j}, b_{j}\right]=1\right\rangle
$$

using surface topology (3.1)-(3.3). The key result of this section is the heredity property of regularity (3.4) and its consequences. (For the cohomology of a group with a single relation, see [11].)

(3.1) Let $X=\{0\} \coprod \coprod_{k=1}^{g}\left(a_{k} \coprod b_{k}\right) \coprod Y$ be a canonical dissection of a compact 
Riemann surface $X$ of genus $g \geq 2$; that is i) $O \in X$ is a point; ii) $a_{k}$ and $b_{k}$ (for $k$ $=1, \ldots, g)$ are simple closed curves on $X$ with base point $O$ disjoint outside $O$ with intersection number $\left\langle a_{i}, b_{j}\right\rangle=-\left\langle b_{j}, a_{i}\right\rangle=\delta_{i j}$ and $\left\langle a_{i}, a_{j}\right\rangle=\left\langle b_{i}, b_{j}\right\rangle=0$ (for $1 \leq i, j \leq g$ ); and iii) $Y$ is an open $4 g$-polygon. We identify $a_{k}$ and $b_{k}$ with the elements of $\pi_{1}(X, O)$ represented by them. This induces the isomorphism: $\Gamma_{g} \simeq \pi_{1}(X, O)$.

(3.2) Let $\mathbf{H} \rightarrow X$ be the holomorphic universal covering of $X$ with $\mathbb{H}$ the complex upperhalf plane. The pull-back of the dissection on $X$ induces a cell decomposition of $\mathbf{H}$. Let $C_{i}$ be the free abelian group generated by the $i$-cells for $i=0,1,2$. Since $\Gamma_{g}$ acts freely on the set of $i$-cells, $C_{i}$ is a $\mathbb{Z} \Gamma_{g}$-free module. Since $\mathbf{H}$ is contractible, the coboundary maps $\partial$ define a free $\mathbb{Z} \Gamma_{g}$ resolution of $\mathbf{Z}$ :

$$
0 \longrightarrow C_{2} \stackrel{\partial_{2}}{\longrightarrow} C_{1} \stackrel{\partial_{1}}{\longrightarrow} C_{0} \stackrel{\varepsilon}{\longrightarrow} \mathbf{Z} \longrightarrow 0
$$

Let us fix a base point $\widetilde{O} \in \mathbf{H}$ over $O \in X$. The 1-cell of $\mathbf{H}$ from $\gamma \widetilde{O}$ to $\delta \widetilde{O}$ (if it exists) is denoted by $[\gamma \tilde{O}, \delta \tilde{O}]=-[\delta \tilde{O}, \gamma \tilde{O}]$. We denote by $Z$ the 2 -cell of $\mathbf{H}$ surrounded by the 1 -cells $\left[R_{i-1} \tilde{O}, R_{i} \tilde{O}\right](1 \leq i \leq 4 g)$, where $R_{i}:=\gamma_{1} \gamma_{2} \cdots \gamma_{i}$ and $\gamma_{1}, \gamma_{2}, \ldots, \gamma_{4 g}$ is the sequence

$$
a_{1}, b_{1}, a_{1}^{-1}, b_{1}^{-1}, a_{2}, b_{2}, a_{2}^{-1}, b_{2}^{-1}, \ldots, a_{g}, b_{g}, a_{g}^{-1}, b_{g}^{-1} .
$$

Using this notation, the complex $\left(C_{*}, \partial\right)(3.2 .1)$ is given explicitly as:

$$
\begin{aligned}
& C_{0}=\mathbb{Z} \Gamma_{g} \cdot \tilde{O}, C_{1}=\bigoplus_{i=1}^{g} \mathbf{Z} \Gamma_{g} \cdot\left[\tilde{O}, a_{i}^{-1} \tilde{O}\right] \oplus \bigoplus_{i=1}^{g} \mathbf{Z} \Gamma_{g} \cdot\left[\tilde{O}, b_{i}^{-1} \tilde{O}\right], C_{2}=\mathbb{Z} \Gamma_{g} \cdot Z \\
& \partial_{1}\left[\tilde{O}, a_{i}^{-1} \tilde{O}\right]=\left(a_{i}^{-1}-1\right) \cdot \tilde{O}, \partial_{1}\left[\tilde{O}, b_{i}^{-1} \tilde{O}\right]=\left(b_{i}^{-1}-1\right) \cdot \tilde{O}, \partial_{2} Z=\sum_{i=1}^{4 g}\left[R_{i-1} \tilde{O},\right. \\
& \left.R_{i} \tilde{O}\right]=\sum_{k=1}^{g} R_{4(k-1)} a_{k} b_{k}\left(\left(a_{k}^{-1}-1\right) \cdot\left[\tilde{O}, b_{k}^{-1} \tilde{O}\right]-\left(b_{k}^{-1}-1\right) \cdot\left[\tilde{O}, a_{k}^{-1} \tilde{O}\right]\right) .
\end{aligned}
$$

It is a direct calculation to check that the following $f_{*}(3.2 .2)$ gives a homotopy equivalence between the complex $\left(C_{*}, \partial\right)(3.2 .1)$ and the standard resolution $F_{*}$ of $\mathbb{Z}$ (see [4, Chap. I.5]).

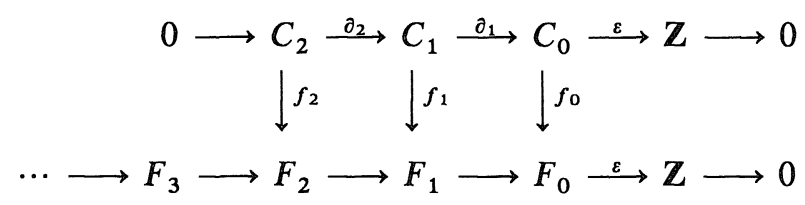

where $f_{0}(\tilde{O}):=[]$,

$$
f_{1}\left(\left[\tilde{O}, a_{i}^{-1} \tilde{O}\right]\right):=\left[a_{i}^{-1}\right] \text { and } f_{1}\left(\left[\tilde{O}, b_{i}^{-1} \tilde{O}\right]\right):=\left[b_{i}^{-1}\right] \text { for } i=1, \ldots, g,
$$




$$
f_{2}(Z):=\sum_{k=1}^{g}\left(\left[R_{4(k-1)} a_{k} b_{k}\left(a_{k}^{-1}-1\right) \mid b_{k}^{-1}\right]-\left[R_{4(k-1)} a_{k} b_{k}\left(b_{k}^{-1}-1\right) \mid a_{k}^{-1}\right]\right) .
$$

(3.3) For a right $\Gamma_{g}$-module $F$, the cohomology group is defined as the homology $H^{*}\left(\Gamma_{g}, F\right) \simeq H^{*}\left(\left(\operatorname{Hom}_{\mathrm{z} \Gamma_{\mathrm{g}}}\left(C_{*}, F\right), \delta^{*}\right)\right)$ of the following complex :

$$
\begin{aligned}
& \operatorname{Hom}_{\mathbf{Z} \Gamma_{\mathbf{g}}}\left(C_{*}, F\right):= \begin{cases}\left.c \in \operatorname{Hom}_{\mathbf{z}}\left(C_{*}, F\right): c\left(\gamma^{-1} \cdot z\right)=c(z) \cdot \gamma \quad{ }^{\forall} \gamma \in \Gamma_{g},{ }^{\forall} z \in C_{*}\right\}\end{cases} \\
& \simeq \begin{cases}F\left(\text { resp. } F^{2 g}, F\right) & \text { if } *=0 \text { (resp. * } * 1, *=2), \\
0\} & \text { otherwise. }\end{cases} \\
& \begin{aligned}
& \delta^{0}(m):=\left(m \cdot\left(a_{i}-1\right), m \cdot\left(b_{i}-1\right)\right)_{i=1}^{g}, \\
& \delta^{1}\left(\left(c\left(a_{i}\right), c\left(b_{i}\right)\right)_{i=1}^{g}\right):=\sum_{k=1}^{g}\left(c\left(b_{k}\right) \cdot\left(a_{k}-1\right)-c\left(a_{k}\right) \cdot\left(b_{k}-1\right)\right)\left(R_{4(k-1)} a_{k} b_{k}\right)^{-1}
\end{aligned} \\
& =\sum_{k=1}^{g}\left(c\left(b_{k}\right) \cdot\left(a_{k}-1\right)-c\left(a_{k}\right) \cdot\left(b_{k}-1\right)\right) \cdot a_{k}^{-1} b_{k}^{-1} \prod_{j=k+1}^{g}\left(a_{j} b_{j} a_{j}^{-1} b_{j}^{-1}\right) .
\end{aligned}
$$

Remark 1. In view of the isomorphisms $Z^{1}\left(\Gamma_{g}, F\right) \simeq Z^{1}\left(\operatorname{Hom}_{\mathbf{z} \Gamma}\left(C_{*}, F\right)\right)$ given by $c \mapsto\left\{c\left(a_{i}\right), c\left(b_{i}\right)\right\}_{i=1}^{g}$, we identify 1 -cocycles for the standard complex and for the complex (3.3.1).

2. It is obvious from (3.3.1) that $H^{j}\left(\Gamma_{g}, F\right)=0$ for $j \geq 3$.

3. Let $F=\mathbf{Z}$ be the infinite cyclic group with trivial $\Gamma_{g}$ action and denote $H^{k}\left(\Gamma_{g}, \mathbb{Z}\right)$ by $H^{k}\left(\Gamma_{g}\right)$ (or by $\left.H^{k}\right)$. The cup product is a skew symmetric form on $H^{1}\left(\Gamma_{g}\right)\left(\simeq \mathbf{Z}^{2 g}\right)$ with values in $H^{2}\left(\Gamma_{g}\right) \simeq \mathbf{Z}$ (see [4]).

(3.4) Let $(\mathscr{S}, l)$ be the complexification (2.1) of an $\mathbf{R}-\Gamma_{g}$ module $R$ for the surface group $\Gamma_{g}(3.1)$ and $\mathscr{F}=\left(F_{i}\right)_{i \geq 0}$ its minimal module (2.3). To calculate the cohomology of $F_{i}$, we introduce a new concept: regularity of $\mathscr{F}$.

Definition 1. Let $i$ be an integer with $i \geq 1$. We say that $\mathscr{F}$ is $i$-regular if the multiplication by $\gamma-1$ on $F_{i}$ is a surjection onto $F_{i-1}$ for all $\gamma \in\left\{a_{1}, b_{1}, \ldots, a_{g}, b_{g}\right\}$.

2. $\mathscr{F}$ is 0 -regular if the following two conditions are satisfied.

i) $\operatorname{rank}_{\mathbf{R}}(\operatorname{ker}(\gamma-1: R \rightarrow R)) \leq 1$ for all $\gamma \in\left\{a_{1}, b_{1}, \ldots, a_{g}, b_{g}\right\}$,

ii) $R=R \cdot\left(a_{i}-1\right)+R \cdot\left(b_{i}-1\right)$ for $i=1, \ldots, 2 g$.

The following heredity of regularity is a key fact throughout the rest of the paper.

Lemma. If $\mathscr{F}$ is $i$-regular, then it is $i+1$-regular for $i \geq 0$.

Proof. We prove this only for $\gamma=a_{1}$. We proceed in 3 steps.

Assertion 1. Assume that $\mathscr{F}$ is $i$-regular. Then the following map $\pi: Z^{1}\left(\Gamma_{g}, F_{i}\right) \longrightarrow F_{i}$, defined by $\pi(c):=c\left(a_{1}\right)$ 
is surjective.

Proof. We understand $Z^{1}\left(\Gamma_{g}, F_{i}\right)$ as the space of the 1-cocycles for the complex (3.3.1) in view of (3.3) Remark 1. One has to show that for any $p_{1} \in F_{i}$ one can find $c=\left\{p_{j}, q_{j}\right\}_{j=1}^{g} \in F_{i}^{2 g}$ satisfying the cocycle condition $\delta^{1}(c)=0$ (3.3.1). This can be solved as follows.

Case $i>0$. Put $c=\left(p_{1}, q_{1}, 0, \ldots, 0\right)$, where $q_{1} \in F_{i}$ is chosen such that $q_{1} \cdot\left(a_{1}-1\right)=p_{1} \cdot\left(b_{1}-1\right) \in F_{i-1}$. This is possible by $i$-regularity assumption.

Case $i=0$. Put $c=\left(p_{1}, 0, p_{2}, q_{2}, \ldots, 0\right)$, where $p_{2}, q_{2} \in F_{0}$ are chosen such that $p_{1} \cdot\left(b_{1}-1\right) a_{1}^{-1} b_{1}^{-1} a_{2} b_{2}=q_{2} \cdot\left(a_{2}-1\right)-p_{2} \cdot\left(b_{2}-1\right) \in F_{0}$.

Assertion 2. Put $Z_{\pi}^{1}\left(\Gamma_{g}, F_{i}\right):=\operatorname{ker}(\pi)$ where $\pi$ is defined in Assertion 1. If $\mathscr{F}$ is $i$-regular, then the natural inclusion $F_{i} \subset \mathscr{S}$ induces a surjection $Z_{\pi}^{1}\left(\Gamma_{g}, F_{i}\right)$ $\rightarrow H^{1}\left(\Gamma_{g}, \mathscr{S}\right)$.

Proof. Case $i=0$. It is enough to show that $\operatorname{Im}\left(Z_{\pi}^{1}\left(\Gamma_{g}, R\right)\right)$ in $H^{1}\left(\Gamma_{g}, R\right)$ $\simeq H^{1}\left(\Gamma_{g}, \mathscr{S}\right)$ has $\mathbf{R}$-codimension $\leq 1$, since then $\operatorname{Im}\left(Z_{\pi}^{1}\left(\Gamma_{g}, F_{0}\right)\right)$ is a $\mathbf{C}$-module containing $\operatorname{Im}\left(Z_{\pi}^{1}\left(\Gamma_{g}, R\right)\right)$ and hence its $\mathbf{C}$-codimension in $H^{1}\left(\Gamma_{g}, \mathscr{S}\right)$ can be at most $[1 / 2]=0$.

For an element $c \in Z^{1}\left(\Gamma_{g}, R\right)$, if $c\left(a_{1}\right) \in R \cdot\left(a_{1}-1\right)$, say $c\left(a_{1}\right)=r \cdot\left(a_{1}-1\right)$ for an $r \in R$, then $c-\delta^{0}(r) \in Z_{\pi}^{1}\left(\Gamma_{g}, R\right)$. Since $\operatorname{codim}_{\mathbf{R}} R \cdot\left(a_{1}-1\right) \leq 1$, this implies that $\delta^{0}(R)+Z_{\pi}^{1}\left(\Gamma_{g}, R\right)$ has $\mathbf{R}$-codimension at most 1 in $Z^{1}\left(\Gamma_{g}, R\right)$. Hence the same holds for the image of $Z_{\pi}^{1}\left(\Gamma_{g}, R\right)$ in $Z^{1}\left(\Gamma_{g}, R\right) / \delta^{0}(R)$.

Case $i>0$. It is enough to show that $Z_{\pi}^{1}\left(\Gamma_{g}, F_{i}\right)+\delta^{0} F_{i}$ is surjective onto $H^{1}\left(\Gamma_{g}, \mathscr{S}\right)$. Let $c$ be any cocycle in $Z^{1}\left(\Gamma_{g}, F_{i-1}\right)$. By the hypothesis, there exists an element $f \in F_{i}$ such that $f \cdot\left(a_{1}-1\right)=\pi(c):=c\left(a_{1}\right) \in F_{i-1}$. Then by definition $c-\delta^{0}(f) \in Z_{\pi}^{1}\left(\Gamma_{g}, F_{i}\right)$. This means $Z^{1}\left(\Gamma_{g}, F_{i-1}\right) \subset Z_{\pi}^{1}\left(\Gamma_{g}, F_{i}\right)+\delta^{0} F_{i}$. Since $Z^{1}\left(\Gamma_{g}, F_{i-1}\right)$ surjects to $H^{1}\left(\Gamma_{g}, \mathscr{S}\right)$, this completes the proof of Assertion 2.

Assertion 3. Surjectivity of the two maps $\pi: Z^{1}\left(\Gamma_{g}, F_{i}\right) \rightarrow F_{i}$ and $Z_{\pi}^{1}\left(\Gamma_{g}, F_{i}\right)$ $\rightarrow H^{1}\left(\Gamma_{g}, \mathscr{S}\right)$ in Assertions 1 and 2 imply the surjectivity of the map: $F_{i+1} \times$ $\left(a_{1}-1\right) \rightarrow F_{i}$ for $i \geq 0$.

Proof. Recalling (2.2.4), we obtain the following diagram: 


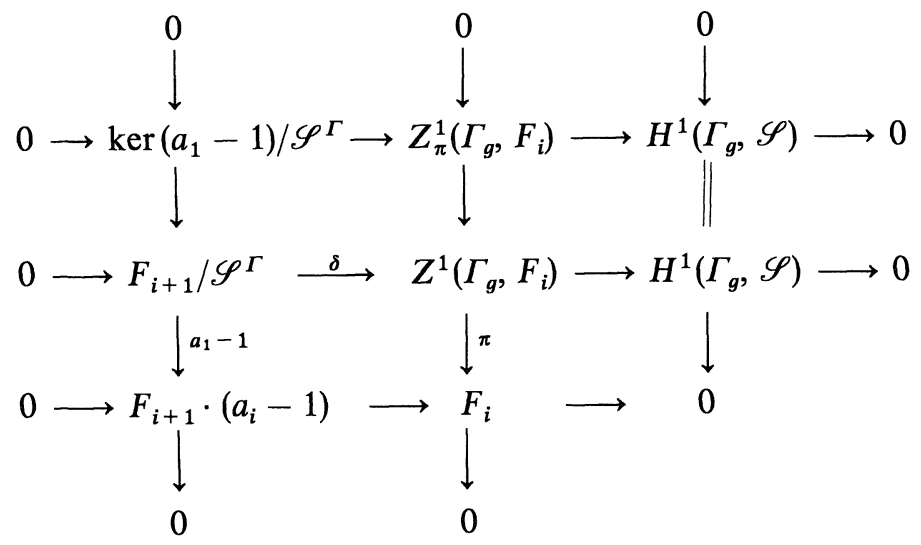

Here all columns and rows except for the last row are exact by the assumptions. Then the last row is also exact.

This completes the proof of the Lemma.

(3.5) An immediate consequence of Lemma (3.4) is the following.

Corollary. Assume that $\mathscr{F}$ is i-regular. Then

$$
\begin{aligned}
& H^{2}\left(\Gamma_{g}, F_{j}\right) \simeq F_{j} / F_{j-1} \quad \text { for } j \geq i . \\
& H^{2}\left(\Gamma_{g}, \mathscr{F}\right)=0 .
\end{aligned}
$$

(Note that $F_{-1}=F_{0}$ in case of 0-regularity. (cf. (2.5.2)).

Proof. Recall the complex (3.3.1) so that $H^{2}\left(\Gamma_{g}, F_{j}\right) \simeq F_{j} / \delta^{1} F_{j}^{2 g}$. If $\mathscr{F}$ is $i$ regular for $i>0$, then the Lemma implies $\delta^{1} F_{j}^{2 g}=F_{j-1}$ for $j \geq i$ so that (3.5.1) holds. Also 0-regularity implies $\delta^{1} F_{0}^{2 g}=F_{0}$ and hence $H^{2}\left(\Gamma_{g}, F_{0}\right)=0$. Taking the inductive limit of (3.5.1), (3.5.2) follows.

(3.6) The coboundary map $\delta^{k}$ (cf. (2.5)).

We show that there is an exact sequence (3.6.4) (cf. (6.3.1)) for a complex defined on the graded pieces of an $i$-regular minimal module $\mathscr{F}$, where the coboundary maps $\delta^{k}$ are defined below. First we put

$$
G_{i}:=F_{i+1} / F_{i}
$$

for $i \geq-1$. Now introduce the coboundary maps

$$
\delta^{k}: G_{i} \otimes_{\mathbf{z}} H^{k}\left(\Gamma_{g}, \mathbf{Z}\right) \longrightarrow G_{i-1} \otimes_{\mathbf{z}} H^{k+1}\left(\Gamma_{g}, \mathbf{Z}\right) \quad \text { for } \quad k=0,1
$$

as follows. Put $\delta^{0}:=\delta$ for the coboundary map $\delta: G_{i} \rightarrow G_{i-1} \otimes_{\mathbf{z}} H^{1}\left(\Gamma_{g}\right)$ introduced in (2.5.1). If $\delta^{k}$ is defined already, then $\delta^{k+1}$ is defined as the composite: 
$G_{i} \otimes H^{k}\left(\Gamma_{g}\right) \stackrel{\delta \times i d}{\longrightarrow} G_{i-1} \otimes H^{1}\left(\Gamma_{g}\right) \otimes H^{k}\left(\Gamma_{g}\right) \stackrel{\text { cup product }}{\longrightarrow} G_{i-1} \otimes H^{k+1}\left(\Gamma_{g}\right)$

In our Fuchsian group case, let us give an explicit formula :

$$
\delta^{1}(\varphi):=\sum_{k=1}^{g}\left(\hat{\varphi}\left(b_{k}\right) \cdot\left(a_{k}-1\right)-\hat{\varphi}\left(a_{k}\right) \cdot\left(b_{k}-1\right)\right) \bmod F_{i-1},
$$

where $\hat{\varphi}(\gamma) \in F_{i+1}$ is an element representing $\varphi(\gamma) \in G_{i}:=F_{i+1} / F_{i}$.

Proof of (3.6.3). Recalling the definition of $\delta$ (2.5.1), we have

$$
(\delta \times i d)(\varphi)(u, v) \equiv \hat{\varphi}(u) \cdot(v-1) \bmod F_{i-1}
$$

for $u, \quad v \in H_{1}\left(\Gamma_{g}\right) \simeq \Gamma_{g} /\left[\Gamma_{g}, \Gamma_{g}\right]$. Let $e_{1}, \ldots, e_{2 g} \in H_{1}\left(\Gamma_{g}\right)$ be a basis and $e^{1}, \ldots, e^{2 g} \in \operatorname{Hom}_{\mathbb{Z}}\left(\Gamma_{g}, \mathbb{Z}\right)$ the dual basis. Then one has an expression

$$
\delta^{1}(\varphi)=\sum_{k, l=1}^{2 g}\left((\delta \times i d)(\varphi)\left(e_{k}, e_{1}\right)\right)\left(e^{k} \vee e^{1}\right) .
$$

By taking the generators $a_{k}, b_{k} \in \Gamma_{g}(1 \leq k \leq g)$ as for representatives of a symplectic basis of $\Gamma_{g} /\left[\Gamma_{g}, \Gamma_{g}\right]$, we obtain the formula.

Lemma. Assume that $\mathscr{F}$ is i-regular. Denote $\mathrm{H}^{k}\left(\Gamma_{g}\right):=\mathbb{H}^{k}\left(\Gamma_{g}, \mathbb{Z}\right)$. Then the following sequences are exact;

$$
0 \longrightarrow G_{j} \stackrel{\delta^{0}}{\longrightarrow} G_{j-1} \otimes_{\mathbb{Z}} H^{1}\left(\Gamma_{g}\right) \stackrel{\delta^{1}}{\longrightarrow} G_{j-2} \otimes_{\mathbb{Z}} H^{2}\left(\Gamma_{g}\right) \longrightarrow 0
$$

for $j>i$. If $i>0$ then the following is also exact:

$$
0 \longrightarrow G_{i} \stackrel{\delta^{0}}{\longrightarrow} G_{i-1} \otimes_{\mathbf{z}} H^{1}\left(\Gamma_{g}\right) \longrightarrow H^{2}\left(F_{i-1}\right) \longrightarrow 0 .
$$

Proof. Suppose $i>0$. For $j \geq i$ the natural map $H^{2}\left(\Gamma_{g}, F_{j}\right) \rightarrow$ $H^{2}\left(\Gamma_{g}, F_{j} / F_{j-1}\right) \simeq F_{j} / F_{j-1} \otimes_{\mathbf{z}} H^{2}\left(\Gamma_{g}\right)$ is bijective, since both modules are isomorphic to $F_{j} / F_{j-1}$ in a natural way (cf. (3.5.1), (3.3.1)). We apply this fact to the sequence (2.5.3), and obtain for $j \geq i$

$$
0 \longrightarrow F_{j+1} / F_{j} \stackrel{\delta^{0}}{\longrightarrow} \operatorname{Hom}_{\mathbf{Z}}\left(\Gamma_{g}, F_{j} / F_{j-1}\right) \longrightarrow H^{2}\left(\Gamma_{g}, F_{j-1}\right) \longrightarrow 0
$$

Let us show that the map $\operatorname{Hom}_{\mathbb{Z}}\left(\Gamma_{g}, F_{j} / F_{j-1}\right) \rightarrow H^{2}\left(\Gamma_{g}, F_{j-1}\right)$ in this sequence is naturally identified with the map $\delta^{1}$ (3.6.2) for $j>i$. This can be checked by comparing the explicit descriptions of the coboundary map $\delta^{1}$ in (3.3.1) and (3.6.3).

If $i=0$, then $\mathscr{F}$ is also 1 -regular. So we apply (3.6.4) for $i=1$. Since $H^{2}\left(F_{0}\right) \simeq F_{0} / \delta\left(F_{0}^{2 g}\right) \simeq 0$ by 0 -regularity, (3.6.5) becomes

$$
0 \longrightarrow G_{1} \stackrel{\delta^{0}}{\longrightarrow} G_{0} \otimes_{\mathbf{z}} H^{1}\left(\Gamma_{g}\right) \longrightarrow 0 .
$$


Since $G_{-1}:=F_{0} / F_{-1}=0$ in the 0 -regular case, (3.6.6) is the initial case of (3.6.4) for $i=0$.

(3.7) The class $\left[G_{i}\right]$ in $K^{0} \mathbf{C}$ ( = the $K$-group of $\mathbf{C}$-vector spaces, see the Remark below) is determined recursively by the use of the sequences (3.6.4) and (3.6.5).

Corollary. Let the setting be as in (3.4). Assume that $\mathscr{F}$ is i-regular. Then the generating function for the sequence $\left[G_{j}\right]$ (for $j \in \mathbf{Z}_{\geq 0}$ ) is a rational function of the form

$$
\sum_{j=0}^{\infty}\left[G_{j}\right] t^{j}=\frac{P(t)}{1-\left[H^{1}\left(\Gamma_{g}, \mathbf{C}\right)\right] \cdot t+\left[H^{2}\left(\Gamma_{g}, \mathbf{C}\right)\right] \cdot t^{2}}
$$

where $P(t)$ is a polynomial of $\mathrm{deg} \leq i$. In particular if $\mathscr{F}$ is 0 -regular,

$$
\sum_{i=0}^{\infty}\left[G_{i}\right] t^{i}=\frac{\left[\overline{H^{1}\left(\Gamma_{g}, \mathscr{S}\right)}\right]}{1-\left[H^{1}\left(\Gamma_{g}, \mathbf{C}\right)\right] \cdot t+\left[H^{2}\left(\Gamma_{g}, \mathbf{C}\right)\right] \cdot t^{2}}
$$

Proof. In the formal power series ring, put

$$
\begin{aligned}
P(t):= & \left(\sum_{j=0}^{\infty}\left[G_{j}\right] t^{j}\right)\left(1-\left[H^{1}\right] \cdot t+\left[H^{2}\right] \cdot t^{2}\right) \\
= & {\left[G_{0}\right]+\left(\left[G_{1}\right]-\left[H^{1}\right] \otimes_{\mathbf{c}}\left[G_{0}\right]\right) t } \\
& \quad+\sum_{j=2}^{\infty}\left(\left[G_{j}\right]-\left[H^{1}\right] \otimes_{\mathbf{c}}\left[G_{j-1}\right]+\left[H^{2}\right] \otimes\left[G_{j-2}\right]\right) \cdot t^{j}
\end{aligned}
$$

where $H^{k}:=H^{k}\left(\Gamma_{g}, \mathbf{C}\right)$ for $k=1,2$. If $i>0$, then (3.6.4) gives the recursion relations: $\left[G_{j}\right]-\left[H^{1}\right] \otimes_{\mathbf{c}}\left[G_{j-1}\right]+\left[H^{2}\right] \otimes_{\mathbf{c}}\left[G_{j-2}\right]=0$ for $j>i$, in the $K$ group, so that $P(t)$ is a polynomial of degree $\leq i$. If $i=0$, then the recursion relations, including (3.6.6), imply that $P(t)$ is a constant, which is the class of $G_{0}$ $:=F_{1} / F_{0}=\overline{H^{1}(\Gamma, \mathscr{S})}(2.4 .2)$.

Remark. There is an isomorphism $K^{0} \mathbf{C} \simeq \mathbf{Z}$ by the correspondence $[G] \mapsto \operatorname{dim}_{\mathbf{C}} G$. Here in the (3.7.1) and (3.7.2), we used $[G]$ rather than $\operatorname{dim}_{\mathbf{C}} G$ for the following reason. In the next paragraph, we study minimal modules associated to Eichler-Shimura isomorphism. The graded pieces $G_{\rho, i}^{n}$ for them depends analytically on the representation $\rho$ of the surface group into $S L(2, \mathbf{R})$. That is, they form vector bundles over the Teichmüller space $T_{g}$ equivariant with the mapping class group action. Then the formula (3.7.1) and (3.7.2) are the formula for the class in the $K$-group (the Grothendieck group) for such vector bundles over $T_{g}$.

\section{§4. The Eichler-Shimura Isomorphism}

We recall the Eichler-Shimura isomorphism (see $[4],[15,16]$ ). The 
minimal modules relative to the isomorphisms are shown to be 0 (or 1-) regular in the sense of (3.4).

(4.1) Let $\operatorname{Sym}^{n}\left(\mathbf{R}^{2}\right)$ be the $n$th symmetric tensor product space over $\mathbb{R}$ of the vector representation space $\mathbb{R}^{2}$ of $S L(2, \mathbf{R})$ for $n \in \mathbb{Z}_{\geq 0}$. The space can be identified with the space of all real polynomials in one variable, say $z$, of degreee $\leq n$. An element $A=\left[\begin{array}{ll}a & b \\ c & d\end{array}\right] \in S L(2, \mathbf{R})$ acts on $\varphi(z) \in S y m^{n}\left(\mathbb{R}^{2}\right)$ on the right :

$$
\varphi(z) \cdot a d^{n / 2}(A):=\varphi\left(\frac{a z+b}{c z+d}\right)(c z+d)^{n} .
$$

(We use the notation $a d^{n / 2}$ for the action, since in case of $n=2$ the action is identified with the adjoint action of $\operatorname{SL}(2, \mathbf{R})$ on $\operatorname{sl}(2, \mathbf{R})$.)

Let $\mathcal{O}_{\mathbf{H}}\left(K_{\mathbf{H}}^{-n / 2}\right):=\mathcal{O}_{\mathbf{H}}\left(\frac{d}{d z}\right)^{n / 2}$ be the invertible sheaf on the upper half plane $\mathbb{H}:=\{z \in \mathbb{C}: \operatorname{Im}(z)>0\}$ with the base $\left(\frac{d}{d z}\right)^{n / 2}$. The element $A=\left[\begin{array}{ll}a & b \\ c & d\end{array}\right] \epsilon$ $S L(2, \mathbf{R})$ acts on the sheaf from the right, denoted again by $a d^{n / 2}$,

$$
\left(\varphi(z)\left(\frac{d}{d z}\right)^{n / 2}\right) \cdot a d^{n / 2}(A):=\varphi\left(\frac{a z+b}{c z+d}\right)(c z+d)^{n}\left(\frac{d}{d z}\right)^{n / 2} .
$$

By definition, the $\mathbf{R}$-linear embedding

$$
\iota_{n}: \operatorname{Sym}^{n}\left(\mathbf{R}^{2}\right) \longrightarrow \Gamma\left(\mathbf{H}, \mathcal{O}_{\mathbf{H}}\left(K_{\mathbf{H}}^{-n / 2}\right)\right), \quad \varphi(z) \longmapsto \varphi(z)\left(\frac{d}{d z}\right)^{n / 2},
$$

for $n \geq 0$ is equivariant with respect to the $a d^{n / 2}$ actions of $S L(2, \mathbb{R})$.

Let $\Gamma_{g}$ be the surface group of genus $g \geq 2$ (3.1.1) and let

$$
\rho: \Gamma_{g} \longrightarrow S L(2, \mathbb{R})
$$

be a faithful discrete and cocompact representation. $\Gamma_{g}$ acts on $\operatorname{Sym}^{n}\left(\mathbb{R}^{2}\right)$ and $\Gamma\left(\mathbf{H}, \mathcal{O}_{\mathbf{H}}\left(K_{\mathbf{H}}^{-n / 2}\right)\right)$ by the composite $a d_{\rho}^{n / 2}:=a d^{n / 2} \circ \rho$, which is equivariant with respect to $l_{n}$.

Remark. 1. $\rho$ induces a representation $\hat{\rho}: \Gamma \rightarrow P S L(2, \mathbb{R})$ and defines a Riemann surface $X:=\hat{\rho}(\Gamma) \backslash H$ of genus $g$. Conversely, for a given $\hat{\rho}$ there exists a lifting $\rho$ (4.1.4), determined up to the choice of spin structure $\in \mathbb{Z}_{2}^{2 g}$ (cf. [17], [2]).

2. The right action of $a d_{\rho}^{n / 2}(\gamma)$ on $\mathcal{O}_{\mathbb{H}}\left(K_{\mathbb{H}}^{-n / 2}\right)$ is equivariant with the left action of $\hat{\rho}(\gamma)^{-1}$ on $\mathbf{H}$ (for $\gamma \in \Gamma_{g}$ ), so the quotient $\mathcal{O}_{\mathbf{H}}\left(K_{\mathbf{H}}^{-n / 2}\right) / \Gamma_{g}$ is an invertible sheaf on the surface $X$, denoted by $\mathcal{O}_{X}\left(K_{X}^{-n / 2}\right)$. The pair $\left(X, \mathcal{O}_{X}\left(K_{X}^{1 / 2}\right)\right)$ is called a spin Riemann surface. Two representations $\rho$ and $\rho^{*}$ give isomorphic spin Riemann surface if and only if they are conjugate in $S L(2, \mathbb{R})$ up to $\operatorname{Aut}\left(\Gamma_{g}\right)$. 
(4.2) The Eichler-Shimura isomorphism is formulated as follows.

Theorem. The map $l_{n}$ induces an isomorphism of $\mathbf{R}$-vector spaces

$$
H_{\rho}^{1}\left(\Gamma_{g}, \operatorname{Sym}^{n}\left(\mathbf{R}^{2}\right)\right) \simeq H_{\rho}^{1}\left(\Gamma_{g}, \Gamma\left(\mathbf{H}, \mathcal{O}_{\mathbf{H}}\left(K_{\mathbf{N}}^{-n / 2}\right)\right)\right.
$$

of the 1 st cohomology group of $\Gamma_{g}$ w.r.t. the action $a d_{\rho}^{n / 2}$.

(The lower script $\rho$ at the notation is added to indicate the dependence of the cohomology group on $\rho$ (4.1.4).)

An outline of the proof [15]. Serre duality implies that the right-hand side of (4.2.1) is dual to $H_{\rho}^{0}\left(\Gamma_{g}, \Gamma\left(\mathbf{H}, \mathcal{O}_{\mathbf{H}}\left(K^{n / 2+1}\right)\right)\right)=$ the space of automorphic forms of weight $n+2$. Then the $\mathbf{R}$-ranks of the both sides of (4.2.1) are shown to be equal in Eichler [4]. The injectivity is shown by Shimura [15] using the Peterson inner product on the automorphic forms.

The isomorphism is generalized to higher-dimensional cases. (See Murakami [12] and the references given there.)

(4.3) The pair $\left(\Gamma\left(\mathbf{H}, \mathcal{O}_{\mathbf{H}}\left(K^{-n / 2}\right), l_{n}\right)\right.$ is a complexification of $S y m^{n}\left(\mathbf{R}^{2}\right)$ in the sense of (2.1) in view of the isomorphism (4.2.1).

The minimal module relative to the pair will be denoted by

$$
\mathscr{F}_{\rho}^{n}=\left(F_{\rho, i}^{n}\right)_{i \geq 0},
$$

which is a filtered $\mathbf{C}-\Gamma_{g}$ submodule of $\Gamma\left(\mathbf{H}, \mathcal{O}\left(K_{\mathbf{H}}^{-n / 2}\right)\right)$ depending on $\rho, n$ and $i$ (cf. (2.3)). Of course, one has the isomorphism: $\operatorname{Sym}^{n}\left(\mathbf{C}^{2}\right) \simeq F_{\rho, 0}^{n}$.

(4.4) The following is a key fact in all what follows.

Lemma. i) The minimal modules $\mathscr{F}_{\rho}^{0}$ for any $\rho$ are 1-regular.

ii) The minimal modules $\mathscr{F}_{\rho}^{n}$ for any $\rho$ and $n>0$ are 0 -regular.

(For the definition of i-regularity, recall (3.4).)

Proof. i) For $f \in \Gamma\left(\mathbf{H}, \mathcal{O}_{\mathbf{H}}\right)$,

$$
\begin{aligned}
f \in F_{\rho, 1}^{0} & \left\langle=>f(\rho(\gamma) z)-f(z)=\text { const } \quad \text { for any } \gamma \in \Gamma_{g}\right. \\
& <=>d \rho(\gamma)^{*}(d f)-d f=0 \quad \text { for any } \gamma \in \Gamma_{g} \\
& <=\rangle:=d f \in \Gamma\left(\mathbf{H}, \Omega_{\mathbf{H}}^{1}\right)^{\rho(\Gamma)} .
\end{aligned}
$$

That is, any $f \in F_{\rho, 1}^{0}$ can be expressed as an indefinite integral $\int^{z} \omega$ for an abelian differential $\omega$ of the first kind on the Riemann surface $X:=\rho\left(\Gamma_{g}\right) \backslash \mathbf{H}$ in such a way that that $f \cdot \gamma-f=\oint_{\gamma} \omega$. The space $\Gamma\left(\mathbf{H}, \Omega_{\mathbf{H}}^{1}\right)^{\rho(\Gamma)} \simeq \Gamma\left(X, \Omega^{1}\right)$ is of rank $g$ over $\mathbf{C}$ and hence $2 g$ over $\mathbf{R}$, and is dual to $H_{1}(X, \mathbf{R})$. Hence for a generator $a_{1}$ of $\Gamma_{g}$ one can find an abelian differential $\omega$ s.t. $\oint_{a_{1}} \omega=1$. 
ii) It is well known that the image $\rho(\gamma) \in S L(2, \mathbf{R})$ of an element $\gamma \neq 1$ of a surface group $\Gamma_{g}$ is hyperbolic, and hence it is conjugate to $A_{r}:=\left[\begin{array}{ll}r & 0 \\ 0 & r^{-1}\end{array}\right]$ for some $r \in \mathbb{R}$ with $|r|>1$. The action of $A_{r}$ on $\operatorname{Sym}^{n}\left(\mathbf{R}^{2}\right)$ is given by $z^{k} \cdot a d^{n / 2}\left(A_{r}\right)$ $=r^{2 k-n} z^{k}$ for $k=0, ., n$. Hence it is semisimple with real eigenvalues $r^{2 k-n}$ (for $0 \leq k \leq n)$. In particular $a d^{n / 2}(A)-1$ has non-trivial kernel if and only if $n$ is even, and then its rank is equal to 1 . For even $n$, let us show that

(*) For any two hyperbolic elements $A$ and $B$ of $S L(2, \mathbf{R})$ the following three conditions i) $\sim$ iii) are equivalent.

i) $A$ and $B$ commute,

ii) $\operatorname{ker}\left(\operatorname{ad}^{n / 2}(A)-1\right)=\operatorname{ker}\left(\operatorname{ad}^{n / 2}(B)-1\right)$,

iii) $\operatorname{im}\left(\operatorname{ad}^{n / 2}(A)-1\right)=\operatorname{im}\left(\operatorname{ad}^{n / 2}(B)-1\right)$.

Proof. We may assume $A=A_{r}$ and $B=A_{s} \cdot A d\left[\begin{array}{ll}a & b \\ c & d\end{array}\right]$. Note that i) is equivalent to "either $a=d=0$ or $b=c=0$ ", since an element of $G L(2)$ commutes with $A_{r}$ if and only if it is diagonal and $A_{s} \cdot A d\left[\begin{array}{ll}a & b \\ c & d\end{array}\right]=A_{s}^{ \pm 1}$ for $a$ $=d=0$ or $b=c=0$. Since the fixed points for $A$ (resp. $B$ ) is spanned by $z^{n / 2}$ (resp. $\left.((a z+b)(c z+d))^{n / 2}\right)$, ii) is possible only when either $b=c=0$ or $a=d$ $=0$. The image for $A$ (resp. $B$ ) is spanned by $z^{k}$ (resp. $\left.(a z+b)^{k}(c z+d)^{n-k}\right)$ for $k=0, \ldots, n, k \neq n / 2$. Hence iii) implies in particular that $(a z+b)^{n}$ and $(c z+d)^{n}$ do not contain the monomial $z^{n / 2}$. This implies again either $a=d=0$ or $b=c$ $=0$. Hence $*$ ) is proved.

(*) implies conditions i) and ii) of (3.4), Lemma 2.

Remark 1. As seen in the proof, the space $F_{\rho, 1}^{0}$ is identified with the space of abelian integrals of the first kind. Generally for $n \geq 0$, an element $\varphi(z)$ $\left(\frac{d}{d z}\right)^{n / 2} \in \Gamma\left(\mathbf{H}, \mathcal{O}_{\mathbf{H}}\left(K^{-n / 2}\right)\right)$ belongs to $F_{\rho, 1}^{n}$ if and only if $\varphi^{(n)}\left(\frac{a z+b}{c z+d}\right)(\mathrm{cz}+d)^{n+2}$ $-\varphi^{(n)}(z)=0$ (5.4.5). Such $\varphi(z)\left(\frac{d}{d z}\right)^{n / 2}$ is called the Eichler integral of weight $n$ [4]. For this reason, we regard elements of $F_{\rho, i}^{n}$ for $i \in \mathbf{N}$ as generalizations of Eichler integrals. In fact $F_{\rho, i}^{n}$ may be regarded as the null space for certain linear differential operators on $X$ (cf. (5.4)).

2. $H^{2}\left(\Gamma_{g}, \operatorname{Sym}^{0}\left(\mathbf{R}^{2}\right)\right)=\mathbf{R}$ and $H^{2}\left(\Gamma_{g}, \operatorname{Sym}^{n}\left(\mathbf{R}^{2}\right)\right)=0$ for $n>0$. (by (3.3.1) and the Lemma.)

(4.5) As consequences of Lemma (4.3), we can apply several result of $\S 3$. For the sake of completeness, we recall and summarize them.

Assertion 1. Put 


$$
G_{\rho, i}^{n}:=F_{\rho, i+1}^{n} / F_{\rho, i}^{n}
$$

for $n \geq 0$ and $i \geq-1$. Then one has the following exact sequences

$$
0 \longrightarrow G_{\rho, j}^{n} \stackrel{\delta^{0}}{\longrightarrow} G_{\rho, j-1}^{n} \otimes_{\mathbf{z}} H^{1}\left(\Gamma_{g}\right) \stackrel{\delta^{1}}{\longrightarrow} G_{\rho, j-2}^{n} \otimes_{\mathbf{z}} H^{2}\left(\Gamma_{g}\right) \longrightarrow 0
$$

for $n \geq 0$ and $j>1$. For $j=1$, we have separate cases.

$$
0 \longrightarrow G_{\rho, 1}^{0} \stackrel{\delta^{0}}{\longrightarrow} G_{\rho, 0}^{0} \otimes_{\mathbf{z}} H^{1}\left(\Gamma_{g}\right) \longrightarrow F_{X, 0}^{0} \otimes_{\mathbf{z}} H^{2}\left(\Gamma_{g}\right) \longrightarrow 0
$$

and

$$
0 \longrightarrow G_{\rho, 1}^{n} \stackrel{\delta^{0}}{\longrightarrow} G_{\rho, 0}^{n} \otimes_{\mathbf{z}} H^{1}\left(\Gamma_{g}\right) \longrightarrow 0 \quad \text { for } n>0 \text {. }
$$

Here we notice that $F_{X, 0}^{0}:=\mathbf{C} \otimes_{\mathbf{R}} R^{0} \simeq \mathbf{C}$.

2. The formula for the cohomology of $F_{\rho, i}^{n}$ in Summary c) can be proven by the formula (2.2.5) and Corollary (3.5).

3. The formula for the generating functions $\sum_{i=0}\left[G_{\rho, i}^{n}\right] \cdot t^{i}$ in Summary f) can be proven by Corollary (3.7).

\section{§5. Local Systems over Spin Riemann Surfaces}

We describe the transformation rules of the filters $F_{\rho, i}^{n}$ of the minimal modules $\mathscr{F}_{\rho}^{n}$ for a representation $\rho$ (4.1.4) by the actions of $\operatorname{PSL}(2, \mathbf{R})$ and $\operatorname{Aut}\left(\Gamma_{g}\right)$. This leads to local systems $F_{X, i}^{n}$ over a spin Riemann surface $\left(X, K_{X}^{1 / 2}\right)$ depending only on the spin class of $\rho$.

(5.1) For $A \in P S L(2, \mathbf{R})$ and a representation $\rho$, we denote by $\rho \cdot \operatorname{Ad}(A)$ the representation given by $\gamma \in \Gamma_{g} \mapsto A^{-1} \rho(\gamma) A \in S L(2, \mathbf{R})$.

Assertion. For given $\rho$ and $n$, the right action $a d^{n / 2}(A)$ of $A \in S L(2, \mathbf{R})$ on $\Gamma\left(\mathbf{H}, \mathcal{O}_{\mathbf{H}}\left(K_{\mathbf{H}}^{-n / 2}\right)\right)$ induces bijections: $\mathscr{F}_{\rho}^{n} \stackrel{\simeq}{\longrightarrow} \mathscr{F}_{\rho \cdot A d(A)}^{n}$ and $F_{\rho, 1}^{n} \stackrel{\cong}{\longrightarrow} F_{\rho \cdot \operatorname{Ad}(A), i}^{n}$ for $i \in \mathbb{Z}_{\geq 0}$. The bijections are equivariant with respect to the action of $\gamma \in \Gamma_{g}$. Hence one has commutative diagrams:

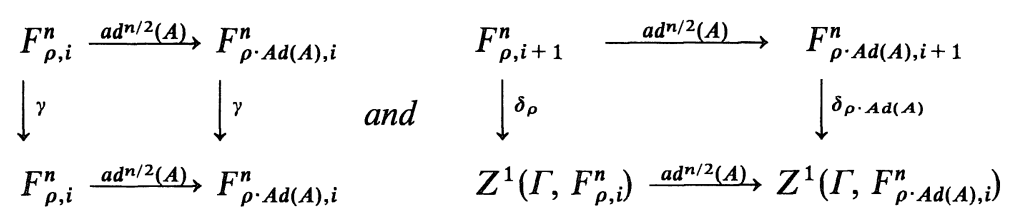

Proof. Recall that the right action of $\gamma \in \Gamma_{g}$ on $\Gamma\left(\mathbf{H}, \mathcal{O}_{\mathbf{H}}\left(K_{\mathbf{H}}^{-n / 2}\right)\right.$ ) (or on $F_{\rho, i}^{n}$ ) is defined by the composition $a d_{\rho}^{n / 2}(\gamma):=a d^{n / 2}(\rho(\gamma))$ (4.1). We verify the following commutativity of the actions on $\Gamma(\mathbf{H}, \Theta)$ : 


$$
a d_{\rho}^{n / 2}(\gamma) \cdot a d^{n / 2}(A)=a d^{n / 2}(A) \cdot a d_{\rho \cdot A d(A)}^{n / 2}(\gamma) .
$$

(Since $\quad a d_{\rho \cdot A d(A)}^{n / 2}(\gamma)=a d^{n / 2}((\rho \cdot A d(A))(\gamma))=a d^{n / 2}\left(A^{-1} \rho(\gamma) A\right)=a d^{n / 2}(A)^{-1} \cdot a d^{n / 2}$ $(\rho(\gamma)) \cdot a d^{n / 2}(A) \stackrel{\doteq}{=} a d^{n / 2}(A)^{-1} \cdot a d_{\rho}^{n / 2}(\gamma) \cdot a d^{n / 2}(A)$.)

By subtracting $a d^{n / 2}(A)$ from both sides of the equality,

$$
\left(a d_{\rho}^{n / 2}(\gamma)-1\right) \cdot a d^{n / 2}(A)=a d^{n / 2}(A) \cdot\left(a d_{\rho \cdot A d(A)}^{n / 2}(\gamma)-1\right) .
$$

This implies that $a d^{n / 2}(A)$ maps $F_{\rho, i}^{n}$ into $F_{\rho \cdot A d(A), i}^{n}$ by induction on $i$, where the case $i=0$ is trivial: $F_{\rho, 0}^{n}=\mathbb{C} \otimes \imath\left(\operatorname{Sym}^{n}\left(\mathbf{R}^{2}\right)\right)$ is invariant under $S L(2, \mathbf{R})$. Since $a d^{n / 2}\left(A^{-1}\right)=\left(a d^{n / 2}(A)\right)^{-1}$, the maps are bijective. The commutativity of diagrams follows from the same relation.

(5.2) An element $\alpha \in \operatorname{Aut}\left(\Gamma_{g}\right)$ acts on a representation $\rho$ on the left by $(\alpha \cdot \rho)(\gamma)$ $:=\rho\left(\alpha^{-1}(\gamma)\right)$. In view of $\rho\left(\Gamma_{g}\right)=(\alpha \cdot \rho)\left(\Gamma_{g}\right)$, we have $\mathscr{F}_{\rho}^{n}=\mathscr{F}_{\alpha \cdot \rho}^{n}$ and $F_{\rho, i}^{n}=\mathscr{F}_{\alpha \cdot \rho, i}^{n}$ for $i, n \geq 0$ as subsets of $\Gamma\left(\mathbf{H}, \mathcal{O}_{\mathbf{H}}\left(K_{\mathbf{H}}^{-n / 2}\right)\right)$.

Assertion. One has the commutative diagrams:

$$
\begin{aligned}
& \begin{array}{llll}
F_{\rho, i}^{n}= & F_{\alpha \cdot \rho, i}^{n} & F_{\rho, i+1}^{n} & =
\end{array} \quad F_{\alpha \cdot \rho, i+1}^{n} \\
& F_{\rho, i}^{n}=F_{\alpha \cdot \rho, i}^{n} \quad Z^{1}\left(\Gamma_{g}, F_{\rho, i}^{n}\right) \stackrel{\alpha *}{\longrightarrow} Z^{1}\left(\Gamma_{g}, F_{\alpha \cdot \rho, i}^{n}\right)
\end{aligned}
$$

where $\alpha_{*}$ is the bijection defined by $\alpha_{*}(c)(\gamma):=c\left(\alpha^{-1}(\gamma)\right)$.

Proof. This follows from the equality

$$
a d_{\alpha \cdot \rho}^{n / 2}(\gamma)=a d^{n / 2}\left(\rho\left(\alpha^{-1}(\gamma)\right)=a d_{\rho}^{n / 2}\left(\alpha^{-1}(\gamma)\right)\right.
$$

(5.3) Define the local system over the Riemann surface $X_{\hat{\rho}}:=\hat{\rho}\left(\Gamma_{g}\right) \backslash \mathbf{H}$,

$$
F_{\rho, i}^{n}:=\Gamma_{g} \backslash\left(\mathbf{H} \times F_{\rho, i}^{n}\right)
$$

by the diagonal action of $\gamma \in \Gamma: \gamma \cdot(z, f):=\left(\hat{\rho}(\gamma) z, f \cdot a d_{\rho}^{n / 2}\left(\gamma^{-1}\right)\right)$.

Lemma. The local system $F_{\rho, i}^{n}\left(n, i \in \mathbb{Z}_{\geq 0}\right)$ depends only on the isomorphism class of the spin Riemann surface $X=\left(X_{\hat{\rho}}, K_{X}^{1 / 2}\right)$.

Proof. Recall that two representations $\rho$ and $\rho^{*}$ give the same spin Riemann surface if and only if there exist $A \in P S L(2, \mathbb{R})$ and $\alpha \in \operatorname{Aut}\left(\Gamma_{g}\right)$ with $\rho^{*}$ $=\alpha \cdot \rho \cdot \operatorname{Ad}(A)$. Then the isomorphism is given by

$$
\begin{aligned}
\psi_{\alpha, A}: X_{\hat{\rho}}:=\hat{\rho}\left(\Gamma_{g}\right) \backslash \mathbf{H} & \simeq X_{\alpha \cdot \hat{\rho} \cdot \operatorname{Ad}(A)}:=(\alpha \cdot \hat{\rho} \cdot \operatorname{Ad}(A))\left(\Gamma_{g}\right) \backslash \mathbf{H} \\
\hat{\rho}\left(\Gamma_{g}\right) \backslash z & \longmapsto(\alpha \cdot \hat{\rho} \cdot \operatorname{Ad}(A))\left(\Gamma_{g}\right) \backslash A^{-1}(z) \\
a d_{\alpha, A}^{n / 2}: \mathcal{O}\left(K_{\mathbf{H}}^{-n / 2}\right) / \rho\left(\Gamma_{g}\right) & \simeq \mathcal{O}\left(K_{\mathbf{H}}^{-n / 2}\right) / \alpha \cdot \rho \cdot \operatorname{Ad}(A)\left(\Gamma_{g}\right) \\
(z, f) & \longmapsto\left(A^{-1}(z), f \cdot a d^{n / 2}(A)\right) .
\end{aligned}
$$


It is enough to show that $a d_{\alpha, A}^{n / 2}$ induces a right $\psi_{\alpha, A}$-isomorphism of the local systems: $*) \quad a d_{\alpha, A}^{n / 2}: F_{\rho, i}^{n} \stackrel{\simeq}{\longrightarrow} F_{\alpha \cdot \rho \cdot A d(A), i}^{n}$ with the property:**) $a d_{\beta \cdot \alpha, A \cdot B}^{n / 2}$ $=a d_{\alpha, A}^{n / 2} \cdot a d_{\beta, B}^{n / 2}$. *) follows from the facts (5.1) and (5.2). **) is also a straightforward calculation and is omitted.

The isomorphism class of local system $F_{\rho, i}^{n}$ over $X$ is denoted

$$
\begin{array}{cc}
F_{X, i}^{n} & \text { for } i, n \in \mathbf{Z}_{\geq 0} \\
\mathscr{F}_{X}^{n}:=\bigcup_{i=0}^{\infty} F_{X, i}^{n} & \text { for } n \in \mathbf{Z}_{\geq 0} .
\end{array}
$$

By construction, the local systems are embedded into the sheaf $\mathcal{O}_{X}\left(K^{-n / 2}\right)$ over $X$ as multi-valued global sections. The graded pieces

$$
G_{X, i}^{n}:=F_{X, i+1}^{n} / F_{X, i}^{n} \quad\left(i, n \in \mathbf{Z}_{\geq 0}\right)
$$

are trivial local systems over $X$, whose ranks (which depend only on $n, i$ and the genus $g$ of $X$ ) are denoted by $g_{g, i}^{n}:=\operatorname{rank}_{\mathbf{C}} G_{X, i}^{n}$. By (4.5) and (3.7), we have

$$
\begin{aligned}
\sum_{i=0}^{\infty} g_{g, i}^{n} t^{i} & =\frac{(g-1)(n+1)}{1-2 g t+t^{2}} \quad \text { for } \quad n>0 \\
& =\frac{g-t}{1-2 g t+t^{2}} \quad \text { for } \quad n=0
\end{aligned}
$$

(5.4) We give an interpretation of the local systems $F_{X, i}^{n}$ as sheaves of solutions of linear differential equations on $X$.

Assertion. There exists a sequence of holomorphic linear differential operators

$$
D_{X, i}^{n}: \mathcal{O}_{X}\left(K_{X}^{d_{g}^{n}, i / 2}\right) \longrightarrow \mathcal{O}_{X}\left(K_{X}^{d_{X}^{n}, i+1 / 2}\right)
$$

of degree $g_{g, i}^{n}$ for $i=0,1,2, \ldots$, such that

i) The local system $F_{X, i}^{n}$ is characterized as the solution of the equation: $D_{X, i}^{n} \circ D_{X, i-1}^{n} \circ \cdots \circ D_{X, 0}^{n}(\phi)=0$. That is,

$$
\operatorname{ker}\left(D_{X, i}^{n} \circ D_{X, i-1}^{n} \circ \cdots \circ D_{X, 0}^{n}\right)=F_{X, i}^{n} .
$$

ii) The map $D_{X, i}^{n} \circ D_{X, i-1}^{n} \circ \cdots \circ D_{X, 0}^{n}$ induces an injection:

$$
D_{X, i}^{n} \circ D_{X, i-1}^{n} \circ \cdots \circ D_{X, 0}^{n}: G_{X, i}^{n} \subset \Gamma\left(X, \mathcal{O}_{X}\left(K_{X}^{d_{X, i+1 / 2}^{n}}\right)\right) .
$$

Here $d_{g, i}^{n}(i \geq 1)$ is given inductively by the formula:

$$
d_{g, 1}^{n}=n+2 \quad \text { and } \quad d_{g, i+1}^{n}=\left(d_{g, i}^{n}+g_{g, i}^{n}\right)\left(g_{g, i}^{n}+1\right) .
$$


Proof. The Lemma is proved using the Wronskian as follows. At the start, for $i=0$, put

$$
D_{X, 0}^{n}\left(\varphi(z)\left(\frac{d}{d z}\right)^{n / 2}\right):=\left(\left(\frac{d}{d z}\right)^{n+1} \varphi(z)\right)(d z)^{(n+2) / 2} .
$$

The following transformation rule can be checked directly:

$$
\left(\frac{d}{d z}\right)^{n+1}\left(\varphi\left(\frac{a z+b}{c z+d}\right)(c z+d)^{n}\right)=\left(\left(\frac{d}{d z}\right)^{n+1} \varphi\right)\left(\frac{a z+b}{c z+d}\right)(c z+d)^{-n-2}
$$

This was studied by G. Bol [3], Peterson and Eichler [4]. i) and ii) for $i=0$ follows obviously from this. Particularly, $D_{X, 0}^{n}$ induces an isomorphism:

$$
D_{X, 0}^{n}: G_{X, 0}^{n} \simeq \Gamma\left(X, \mathcal{O}_{X}\left(K_{X}^{n / 2+1}\right)\right)
$$

Suppose the operators $D_{X, 0}^{n}, \ldots, D_{X, i-1}^{n}$ for $i>0$ are constructed. Let $\varphi_{j}(z)(d z)^{d_{i} / 2} \in D_{X, i-1}^{n} \circ D_{X, i-2}^{n} \circ \cdots \circ D_{X, 0}^{n}\left(F_{p, i}^{n}\right)$ (for $1 \leq j \leq g_{i}:=g_{g, i}^{n}$ ), be a $\mathbb{C}$-basis for the image of $G_{\rho, i}^{n}$. For $\varphi(z)(d z)^{d_{z} / 2} \in \mathcal{O}_{X}\left(K_{X}^{d_{1} / 2}\right)$, put

$$
D_{X, i}^{n}\left(\varphi(z)(d z)^{d_{i} / 2}\right):=\left|\begin{array}{ccc}
\varphi_{1}(z), & \varphi_{2}(z), \ldots, \varphi_{g_{i}}(z), \quad \varphi(z) \\
\varphi_{1}^{\prime}(z), & \varphi_{2}^{\prime}(z), \ldots, \quad \varphi_{g_{i}}^{\prime}(z), \quad \varphi^{\prime}(z) \\
\varphi_{1}^{(2)}(z), & \varphi_{2}^{(2)}(z), \ldots, \varphi_{g_{i}}^{(2)}(z), \varphi^{(2)}(z) \\
\cdots \cdots, & \ldots \cdots, \cdots, \cdots \cdots, \cdots \cdots \\
\varphi_{1}^{\left(g_{i}\right)}(z), & \varphi_{2}^{\left(g_{i}\right)}(z), \cdots, \varphi_{g_{i}}^{\left(g_{i}\right)}(z), \varphi^{\left(g_{i}\right)}(z)
\end{array}\right|(d z)^{d_{i+1} / 2}
$$

(Here in this definition, there is an ambiguity of a choice of the basis of $G_{X, i}^{n}$. See the following Remark 1.)

$D_{X, i}^{n}$ commutes with the adjoint action of the $\Gamma_{g}$. To see this, for $A=\left[\begin{array}{ll}a & b \\ c & d\end{array}\right] \in \rho\left(\Gamma_{g}\right), \quad$ substitute $\varphi_{1}(A(z))(c z+d)^{-d_{i}}, \ldots, \varphi_{g_{\mathrm{v}}}(A(z))(c z+d)^{-d_{i}}$, $\varphi(A(z))(c z+d)^{-d_{i}}$ for $\varphi_{1}(z), \ldots, \varphi_{g_{1}}(z), \varphi(z)$ in the definition of $D_{X, i}^{n}$. Apply the fact that $\left(\frac{d}{d z}\right)^{k}\left(\varphi(A(z))(c z+d)^{-d}\right)=\varphi^{(k)}(A(z))(c z+d)^{-d-2 k}+$ $\sum_{j=1}^{k} c_{j} \varphi^{(k-j)}(A(z))(c z+d)^{-d-2 k+j}$ for some constants $c_{j}$, so that the right hand is equal to

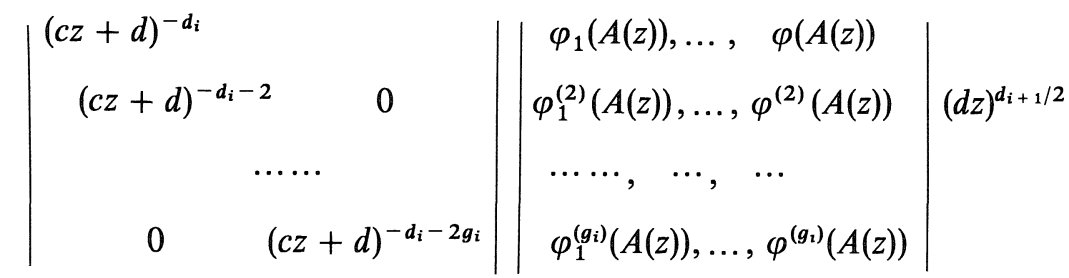

$$
\begin{aligned}
& =D_{X, i}^{n}\left(\varphi(z)(d z)^{d_{i} / 2}\right) \cdot a d^{-d_{i+1} / 2}(A) \text { for } \sum_{j=0}^{g_{i}}\left(d_{i}+2 j\right)=d_{i+1} \text {. On the other hand, }
\end{aligned}
$$


$\varphi_{j}(z)(d z)^{d_{i} / 2} \in \Gamma\left(\mathbf{H}, \mathcal{O}_{\mathbf{H}}\left(K^{d_{i} / 2}\right)\right)^{\Gamma_{\mathbf{g}}}$ means $\varphi_{j}(A(z))(c z+d)^{-d_{i}}=\varphi_{j}(z)$ for $1 \leq j \leq g_{i}$. This implies that

$$
D_{X, i}^{n}\left(f \cdot a d_{\rho}^{-d_{i} / 2}(\gamma)\right)=D_{X, i}^{n}(f) \cdot a d_{\rho}^{-d_{i+1 / 2}}(\gamma)
$$

for $f \in \mathcal{O}_{\mathbf{H}}\left(K_{\mathbf{H}}^{-d_{i} / 2}\right)$ and $\gamma \in \Gamma_{g}$ as a generalization of Bol's relation. So the operator is well defined. By properties of the Wronskian, i) is verified. To prove ii), observe that $f \in \Gamma\left(\mathbf{H}, \mathcal{O}\left(K_{\mathbf{H}}^{-n / 2}\right)\right)$ belongs to $F_{\rho, i+1}^{n}$ if and only if $f$ $-f \cdot a d_{\rho}^{n / 2}(\gamma) \in F_{\rho, i}^{n}$. Applying $D_{X, i}^{n} \circ \cdots \circ D_{X, 0}^{n}$ to this relation, we obtain

$$
D_{X, i}^{n} \circ \cdots \circ D_{X, 0}^{n}(f)-D_{X, i}^{n} \circ \cdots \circ D_{X, 0}^{n}(f) \cdot a d_{\rho}^{-d_{i+1} / 2}=0 .
$$

Remark 1. To be more precise, the operator $D_{X, i}^{n}$ must be normalized as $\frac{1}{\operatorname{det}\left(G_{X, i}^{n}\right)} D_{X, i}^{n}$ to kill the ambiguity arising from the choice of basis of $G_{X, i}^{n}$. For the purpose, one needs to study the vector bundles $\bigcup_{[x] \in \mathscr{T}_{\mathrm{g}}}\left(G_{X, i}^{n}\right)$ over the moduli space $\mathscr{T}_{g}$ of curves of genus $g$. This is beyond the treatment in this paper.

2. The isomorphisms $\overline{F_{\rho, 1}^{n} / F_{\rho, 0}^{n}} \simeq H^{1}\left(X, \mathcal{O}_{X}\left(K_{X}^{-n / 2}\right)(2.4 .2)\right.$ and $D_{X, 0}^{n}: F_{\rho, 1}^{n} / F_{\rho, 0}^{n}$ $\simeq \Gamma\left(X, \mathcal{O}_{X}\left(K_{X}^{n / 2+1}\right)\right)(5.4 .5)$, and Serre duality between $H^{1}\left(X, \mathcal{O}_{X}\left(K_{X}^{-n / 2}\right)\right)$ and $\Gamma\left(X, \mathcal{O}_{X}\left(K_{X}^{n / 2+1}\right)\right)$ implies that the space $G_{\rho, 1}^{n}:=F_{\rho, 1}^{n} / F_{\rho, 0}^{n}$ and its conjugate are $\mathbf{C}$ dual of each other. This is nothing but the Weil-Petersson metric on the space.

\section{§6. Algebra Structure on $\mathscr{H}_{X}$}

We introduce an algebra structure on $\mathscr{H}_{X}:=\bigoplus_{n=0}^{\infty} \mathscr{F}_{X}^{n} / F_{X, 0}^{n}$, whose associated graded ring $\mathscr{G}_{X}:=\operatorname{gr}\left(\mathscr{H}_{X}\right)$ admits derivations by the elements of $\Gamma_{g}$.

(6.1) Recall Bol's map $D_{X, 0}^{n}:=\partial^{n+1}: \Gamma\left(\mathbf{H}, \mathcal{O}\left(K^{-n / 2}\right)\right) \rightarrow \Gamma\left(\mathbf{H}, \mathcal{O}\left(K^{n / 2+1}\right)\right)(5.4)$ on global sections. Obviously $\partial^{n+1}$ is surjective and equivariant with respect to the action of $\Gamma_{g}$. Its kernel is $F_{0}^{n}$.

Assertion. The product $K_{\mathbf{H}}^{n / 2+1} \times K_{\mathbf{H}}^{m / 2+1} \rightarrow K_{\mathbf{H}}^{(n+m) / 2+2}$ on the tensors of the halfcanonical bundle $K_{\mathbf{H}}^{1 / 2}$ induces a $\Gamma_{g}$-equivariant and filter preserving product map:

$$
\begin{aligned}
\partial^{n+1}\left(\mathscr{F F}_{X}^{n}\right) & \times \partial^{m+1}\left(\mathscr{F}_{X}^{m}\right) \longrightarrow \partial^{n+m+3}\left(\mathscr{F}_{X}^{n+m+2}\right) . \\
\partial^{n+1}\left(F_{X, i+1}^{n}\right) & \times \partial^{m+1}\left(F_{X, j+1}^{m}\right) \longrightarrow \partial^{n+m+3}\left(F_{X, i+j+1}^{n+m+2}\right)
\end{aligned}
$$

for $i, j \geq-1$.

Proof. We have only to show that the image of the filters by the product map belongs to the filter described in (6.1.1)* We proceed by induction on $i$ 
and $j$. First we remark that:

for an element $\varphi \in \Gamma\left(\mathbf{H}, \mathcal{O}\left(K^{n / 2+1}\right)\right)$ and for $i \in \mathbb{Z}_{\geq 0}$, we have $\varphi \in \partial^{n+1}\left(F_{X, i+1}^{n}\right)$ if and only if $\varphi \cdot a d_{\rho}^{-n / 2-1}(\gamma)-\varphi \in \partial^{n+1} F_{X, i}^{n}$ for all $\gamma \in \Gamma_{g}$.

If $i=-1$ (resp. $j=-1$ ), then $\partial^{n+1}\left(F_{X, 0}^{n}\right)$ (resp. $\left.\partial^{m+1}\left(F_{X, 0}^{m}\right)\right)$ is 0 so that the image of the map is $0 \subset \partial\left(F_{X, 0}^{n+m+2}\right)$. Now suppose $i, j \geq 0$ and take elements $\varphi \in \partial^{n+1}\left(F_{X, i+1}^{n}\right)$ and $\left.\phi \in \partial^{m+1}\left(F_{X, j+1}^{m}\right)\right)$. Then for $\gamma \in \Gamma_{g}$,

*) $\quad(\varphi \cdot \phi) a d_{\rho}(\gamma)-\varphi \cdot \phi$

$$
=\left(\varphi \cdot a d_{\rho}(\gamma)-\varphi\right) \cdot \phi+\varphi \cdot\left(\phi \cdot a d_{\rho}(\gamma)-\phi\right)+\left(\varphi \cdot a d_{\rho}(\gamma)-\varphi\right) \cdot\left(\phi \cdot a d_{\rho}(\gamma)-\phi\right),
$$

which belongs to $\partial^{n+m+3}\left(F_{X, i+j}^{n+m+2}\right)$ by hypothesis. By the above remark, $\varphi \cdot \phi$ belongs to $\partial^{n+m+3}\left(F_{X, i+j+1}^{n+m+2}\right)$.

Corollary. The product (6.1.1) induces a product $G_{X, i}^{n} \times G_{X, j}^{m} \rightarrow G_{X, i+j}^{n+m+2}$ for $i, j, n, m \geq 0$. For $\gamma \in \Gamma_{g}$, let $\delta_{\gamma}$ be the coboundary map defined in (2.5.1). Then

$$
\delta_{\gamma}(\varphi \cdot \phi)=\delta_{\gamma}(\varphi) \cdot \phi+\varphi \cdot \delta_{\gamma}(\phi)
$$

for $\varphi \in G_{X, i}^{n}, \phi \in G_{X, j}^{m}$ and $\gamma \in \Gamma_{g}$. Here $G_{X, i}^{n}:=F_{X, i+1}^{n} / F_{X, i}^{n}$ cf. (3.6.1).

(6.2) Inspired by the calculations in (6.1), we introduce:

$$
\mathscr{H}_{X}:=\bigoplus_{n=0}^{\infty} \mathscr{F F}_{X}^{n} / F_{X, 0}^{n} \simeq \bigoplus_{n=0}^{\infty} \partial^{n+1} \mathscr{F}_{X}^{n}
$$

Each summand will be denoted as

$$
\mathscr{H}_{X}^{n}:=\mathscr{F}_{X}^{n} / F_{X, 0}^{n} \simeq \partial^{n+1} \mathscr{F}_{X}^{n}
$$

this carries a filtration

$$
H_{X, i}^{n}:=F_{X, i+1}^{n} / F_{X, 0}^{n} \simeq \partial^{n+1} F_{X, i+1}^{n} \quad i=0,1,2, \ldots
$$

induced by that on $\mathscr{F}_{X}^{n}$. Then $(6.1 .1)^{*}$ can be rewritten as a product:

$$
H_{X, i}^{n} \times H_{X, j}^{m} \longrightarrow H_{X, i+j}^{n+m+2}
$$

and so $\mathscr{H}_{X}$ is a graded algebra with increasing filtration

$$
\mathscr{H}_{X, i}:=\bigoplus_{n=0}^{\infty} H_{X, i}^{n}, \quad i=0,1,2, \ldots
$$

The first filter: $\mathscr{H}_{X, 0} \simeq \bigoplus_{n=0}^{\infty} F_{X, 1}^{n} / F_{X, 0}^{n} \simeq \bigoplus_{n=0}^{\infty} \Gamma\left(\mathbf{H}, \mathcal{O}_{\mathbf{H}}\left(K_{\mathbf{H}}^{n / 2+1}\right)\right)^{\Gamma_{\mathbf{g}}}$ is (a part of) the half canonical ring for the spin Riemann surface $\left(X, K_{X}^{1 / 2}\right)$ associated to the representation $\rho$. It is well known that $\mathscr{H}_{X, 0}$ is a finitely generated algebra over $\mathrm{C}$ and is noetherian.

In the remaining of this paragraph, we prove the following: 
Lemma. $\mathscr{H}_{X}$ is an integral domain over $\mathscr{H}_{X, 0}$ such that each filter $\mathscr{H}_{X, i}$ is a finite module over $\mathscr{H}_{\mathrm{X}, 0}$.

By generalities in commutative algebra, to prove the Lemma, it is enough to prove the same statement (Lemma* below) for the graded algebra $\operatorname{gr}\left(\mathscr{H}_{X}\right)$ $:=\bigoplus_{i=0}^{\infty} \mathscr{H}_{X, i} / \mathscr{H}_{X, i-1}\left(\right.$ Here $\left.\mathscr{H}_{X,-1}:=0\right)$. The associated graded algebra of $\mathscr{H}_{X}$, which we denote by $\mathscr{G}_{X}$, is given by

$$
\mathscr{G}_{X}:=\operatorname{gr}\left(\mathscr{H}_{X}\right)=\bigoplus_{i=0}^{\infty} \mathscr{G}_{X, i},
$$

where $\mathscr{G}_{X, i}:=\mathscr{H}_{X, i} / \mathscr{H}_{X, i-1}=\bigoplus_{n=0}^{\infty} F_{X, i+1}^{n} / F_{X, i}^{n}=\bigoplus_{n=0}^{\infty} G_{X, i}^{n}(i \geq 0)$. Of course $\mathscr{G}_{X}$ is a bigraded algebra, as $G_{X, i}^{n} \times G_{X, j}^{m} \rightarrow G_{X, i+j}^{n+m+2}$ is induced from (6.1.1). The 0graded part $\mathscr{G}_{X, 0}$ w.r.t. the index $i$ is the same as the $\mathscr{H}_{X, 0}$. Therefore we prove the following:

\section{Lemma* i) $\mathscr{G}_{X}$ is an integral domain over $\mathscr{G}_{X, 0}$. \\ ii) For $i \geq 0, \mathscr{G}_{X, i}$ is a $\mathscr{G}_{X, 0}$-finite module.}

(6.3) To prove the Lemma*, we summarize some of the previous results in a Theorem, which describes a resolution of the half canonical ring $\mathscr{G}_{X, 0}$; this can be regarded as the main result of the present paper.

Theorem. Let $\delta^{k}: \mathscr{G}_{X, i} \otimes H^{k}\left(\Gamma_{g}, \mathbf{Z}\right) \rightarrow \mathscr{G}_{X, i-1} \otimes H^{k+1}\left(\Gamma_{g}, \mathbf{Z}\right)$ be the coboundary map introduced in (3.6.2) for $i \geq 0$ and $k=0,1$. Then they are $\mathscr{G}_{X, 0^{-}}$ module homomorphisms, and they define the following resolution of the halfcanonical ring $\mathscr{G}_{X, 0}$ :

$$
0 \longrightarrow \mathscr{G}_{X, 0} \longrightarrow \mathscr{G}_{X} \stackrel{\delta^{0}}{\longrightarrow} \mathscr{G}_{X} \otimes_{\mathbf{z}} H^{1}\left(\Gamma_{g}\right) \stackrel{\delta^{1 \otimes l}}{\longrightarrow}\left(\mathscr{G}_{X} \oplus F_{X, 0}^{0}\right) \otimes_{\mathbf{z}} H^{2}\left(\Gamma_{g}\right) \longrightarrow 0 .
$$

Here $F_{X, 0}^{0} \simeq \mathbf{C}$ as a $\mathscr{G}_{X, 0}$-module is annihilated by any element of $\mathscr{G}_{X, 0}$. The map $\imath$ to the factor $F_{X, 0}^{0}$ is given by abelian integrals:

$$
f \in \operatorname{Hom}_{\mathbf{Z}}\left(\Gamma_{g}, G_{X, 0}^{0}\right) \longrightarrow \sum_{i=1}^{g} \int_{b_{i}} f\left(a_{i}\right)-\int_{a_{i}} f\left(b_{i}\right) \in F_{X, 0}^{0} \simeq \mathbf{C} .
$$

Proof. The exactness of (6.3.1) follows from Lemma (4.3) and Lemma (3.6). The fact that $\delta^{0}$ and $\delta^{1}$ are commutative with the $\mathscr{G}_{X, 0}$-module structure follows from (6.1.2). The map $\imath$ comes from the exact sequence (3.6.5) for $i=1$ and $n=0$ :

$$
0 \longrightarrow G_{X, 1}^{0} \longrightarrow G_{X, 0}^{0} \otimes_{\mathbf{Z}} H^{1}\left(\Gamma_{g}, \mathbf{Z}\right) \stackrel{\iota}{\longrightarrow} F_{X, 0}^{0} \otimes_{\mathbf{Z}} H^{2}\left(\Gamma_{g}, \mathbf{Z}\right) \longrightarrow 0
$$

To obtain an explicit formula (6.3.2), we refer to (3.6.3) and its proof. 
Remark. The exactness of (6.3.1) can be reformulated in terms of the cohomology of the complex $\left(\mathscr{G}_{X} \otimes_{\mathbf{Z}} H^{*}, \delta^{*}\right)$ defined by

$$
H^{k}\left(\mathscr{G}_{X} \otimes_{\mathrm{Z}} H^{\cdot}\left(\Gamma_{g}\right), \delta^{\cdot}\right)= \begin{cases}\mathscr{G}_{X, 0} & k=0 \\ F_{X, 0}^{0} & k=1, \\ 0 & \text { otherwise. }\end{cases}
$$

(6.4) Recall the coboundary map $\delta_{\gamma}: G_{X, i+1}^{n} \rightarrow G_{X, i}^{n}$ for $\gamma \in \Gamma_{g}$ (2.5). The relation (6.1.2) implies that $\delta_{\gamma}$ is a derivation of the algebra $\mathscr{G}_{X}$ over $\mathscr{G}_{X, 0}$. By a use of derivations, one can recover the filtration on $\mathscr{G}_{X}$ as follows:

for an element $f \in \mathscr{G}_{X}$, the following are equivalent

i) $f \in \bigoplus_{i=0}^{d} \mathscr{G}_{X, i}$, ii) $\delta_{1} \cdots \delta_{d+1} f=0$ for all $\delta_{1}, \ldots, \delta_{d+1} \in \operatorname{Der}\left(\mathscr{G}_{X} / \mathscr{G}_{X, 0}\right)$.

Proof. ii) implies i) by the injectivity of $\delta^{0}$ in (3.6.4). i) implies ii) because the derivations have degree -1 by definition.

Proof of Lemma* i). It is enough to show that the product:

$$
\mathscr{G}_{X, i} \times \mathscr{G}_{X, j} \longrightarrow \mathscr{G}_{X, i+j}
$$

does not have zero-divisors. Let us prove this by induction on $i$ and $j$. The start of the induction: when $i=j=0$, the above product is just the product in the half canonical ring $\mathscr{G}_{X, 0}$ which is obviously integral.

Take $f \in \mathscr{G}_{\boldsymbol{X}, \boldsymbol{i}}$ for $i \geq 1$, and $g \in \mathscr{G}_{\boldsymbol{X}, 0}$ and assume $f g=0$. For any derivation $\delta$, one has $0=\delta(f g)=\delta(f) g+f \delta(g)=\delta(f) g$. Hence either $g=0$ or $\delta(f)=0$ for any $\delta$ by induction on $i$. Hence either $g=0$ or $f=0$.

Let $i, j \geq 1$ and take $f \in \mathscr{G}_{X, i}$ and $g \in \mathscr{G}_{X, j}$ with $f \neq 0, g \neq 0$. Suppose $f g$ $=0$. Let $\delta$ be any derivation. If $\delta^{i} f \neq 0$, then let us show $\delta^{j-k} g=0$ for $k=$ $-1,0,1, \ldots, j$ by induction on $k$, where the case of $k=-1$ is clear. Assume true for $k-1$. Then the relation $0=\delta^{i+j-k}(f g)=\left(\begin{array}{c}i+j-k \\ i\end{array}\right)\left(\delta^{i} f\right)\left(\delta^{j-k} g\right)$ implies $\delta^{j-k} g=0$ by induction. Hence $g=0$ for $k=j$ contradicts the assumption on $g$. Thus $\delta^{i} f=0$, and similarly $\delta^{j} g=0$. If $i-1>0$ and $\delta^{i-1} f \neq 0$, then again a similar argument shows that $\delta^{j-1-k} g=0$ for $k=$ $-1,0, \ldots, j-1$, which gives a contradiction. This implies again that $\delta^{i-1} f=0$ and also $\delta^{j-1} g=0$ as far as $i-1>0$ and $j-1>0$. Repeating a similar argument for $p$ inductively, we obtain $\delta^{i-p} f=0$ and $\delta^{j-p} g=0$ for $0 \leq p$ $<\min (i, j)$. By assuming $i \leq j$, this implies that $\delta f=0$ for any derivation $\delta$ of degree -1 and hence $f=0$.

ii) The exact sequence (6.3.1) implies the exact sequences:

$$
0 \longrightarrow \mathscr{G}_{X, 1} \stackrel{\delta^{0}}{\longrightarrow} \mathscr{G}_{X, 0} \otimes_{\mathrm{Z}} H^{1} \stackrel{\delta^{1}}{\longrightarrow} G_{X,-1}^{0} \otimes_{\mathrm{Z}} H^{2} \longrightarrow 0
$$


and

$$
0 \longrightarrow \mathscr{G}_{X, i+1} \stackrel{\delta^{0}}{\longrightarrow} \mathscr{G}_{X, i} \otimes_{\mathbf{Z}} H^{1} \stackrel{\delta^{1}}{\longrightarrow} \mathscr{G}_{X, i-1} \otimes_{\mathbf{Z}} H^{2} \longrightarrow 0 \quad(i>0)
$$

as $\mathscr{G}_{X, 0}$-module. Since $\mathscr{G}_{X, 0}$ is noetherian, by induction on $i$, this implies the finiteness of $\mathscr{G}_{X, i}$ as $\mathscr{G}_{X, 0}$-module for $i \geq 0$.

Remark. The ring $\mathscr{G}_{X}$ is neither noetherian nor of finite Krull dimension. (The denominator $1-2 g t+t^{2}$ of the dimension formula (5.3.3) has a real root $t_{0}=g-\sqrt{g^{2}-1}$ such that $0<t_{0}<1$. This implies the exponential growth $g_{g, i}^{n} \sim O\left(t_{0}^{-i}\right)$ of the ranks of $G_{X, i}^{n}$ in $i$ so that the graded ring can not be noetherian.

\section{Appendix}

In the Appendix, for a 0-regular minimal module $\mathscr{F}=\left(F_{i}\right)_{i \geq 0}$ for a surface group $\Gamma_{g}$, we give a $\mathbf{Z}$-structure on the $\mathrm{C}$-vector spaces $G_{i}:=F_{i+1} / F_{i}$ (for $i \geq 0$ ). This is done by an explicit lattice description of the $G_{i}$ 's (see (A.5)).

We introduce a sequence of lattices $L_{n}$ and maps $\delta_{n}^{0}: L_{n+1} \rightarrow \operatorname{Hom}_{\mathbf{Z}}\left(\Gamma_{g}, L_{n}\right)$ $\simeq H \otimes_{\mathbf{z}} L_{n}$ (for $\left.n \geq 0\right)$ by induction on $n$. (Here $H:=\operatorname{Hom}_{\mathbf{Z}}\left(\Gamma_{g}, \mathbf{Z}\right)$ is the symplectic lattice together with the symplectic form $I$.) The induction starts with $L_{0}:=\mathbf{Z}, L_{1}:=H$ and $\delta_{0}^{0}: L_{1} \rightarrow H \otimes_{\mathbf{z}} \mathbf{Z}$ the natural isomorphism. Suppose that $L_{n}$ and $\delta_{n-1}^{0}$ are already defined for some $n>0$; define the map $\delta_{n}^{1}: H \otimes_{\mathrm{z}} L_{n}$ $\rightarrow L_{n-1}$ as the composite $H \otimes_{\mathbf{z}} L_{n} \stackrel{i d \times \delta^{0}}{\longrightarrow} H \otimes_{\mathbf{z}} H \otimes_{\mathbf{z}} L_{n-1} \stackrel{I \times i d}{\longrightarrow} L_{n-1}$. Then we put

$$
\begin{aligned}
L_{n+1} & :=\operatorname{ker}\left(\delta_{n}^{1}\right), \\
\delta_{n}^{0} & :=\text { the canonical inclusion map of } L_{n+1} \text { into } H \otimes_{\mathrm{z}} L_{n} .
\end{aligned}
$$

Thus one has the following exact sequence:

$$
0 \longrightarrow L_{n+1} \stackrel{\delta_{n}^{0}}{\longrightarrow} H \otimes_{\mathrm{z}} L_{n} \stackrel{\delta_{n}^{1}}{\longrightarrow} L_{n-1} \quad(n \geq 0) \text {. }
$$

(Here by convention, we set $L_{-1}=0$.)

Lemma 1. For $p, q \geq 0$, one can define a contraction map $l_{p q}: L_{p} \otimes_{\mathbf{z}} L_{q}$ $\rightarrow L_{p-1} \otimes L_{q-1}$ in term of the cup product $I$, giving rise to an exact sequence:

$$
0 \longrightarrow L_{p+q} \longrightarrow L_{p} \otimes_{\mathbf{z}} L_{q} \stackrel{i_{p q}}{\longrightarrow} L_{p-1} \otimes_{\mathbf{z}} L_{q-1} \longrightarrow 0
$$

2. There exists an integral bilinear form $I_{n}$ on $L_{n}(n \geq 0)$, symmetric or skewsymmetric according as $n$ is even or odd, and compatible with the inclusions $L_{p+q}$ 
$\rightarrow L_{p} \otimes_{\mathbf{z}} L_{q}$ for $p, q \geq 0$.

Proof. Rewriting the definition of $L_{n}$ inductively, one has the following description of $L_{n}$ for $n \geq 1$.

$$
L_{n} \simeq\left\{\varphi \in H^{\otimes n}: I_{j}(\varphi)=0 \quad \text { for } \quad j=1, \ldots, n-1 .\right\}
$$

where $H^{\otimes n}$ denotes the tensor product of $n$ copies of $H$ over $\mathbf{Z}$ and $I_{j}$ is the contraction map from $H^{\otimes n}$ to $H^{\otimes(n-2)}$ defined by the cup product $I$ of $j$ th and $(j+1)$ th components for $1 \leq j \leq n-1$.

1. The restriction of the linear form $I_{p}$ on $H^{\otimes(p+q)}$ induces the map $l_{p q}$ on $L_{p} \otimes_{\mathbf{z}} L_{q}$ whose range is $L_{p-1} \bigotimes_{\mathbf{z}} L_{q-1} \subset H^{\otimes(p+q-2)}$ and whose kernel is $L_{p+q}$. The surjectivity of $l_{p q}$ will be shown by induction on $p$. If $p=0$, this is trivial since $L_{-1}=0$ and $L_{0}=\mathbf{Z}$. For $p=1$, we put $l_{q}:=l_{1 q}$ and prove the surjectivity of $l_{q}(q \geq 0)$ as follows.

Asserion. Let $e_{1}, e_{2}, f_{1}, f_{2}$ be elements of $H$ with the properties: $I\left(e_{1}, e_{2}\right)$ $=I\left(e_{2}, e_{1}\right)=0$ and $I\left(e_{1}, f_{1}\right)=I\left(e_{2}, f_{2}\right)=1$. Then the restriction of $l_{q}: e_{i} \otimes L_{q}$ $\rightarrow L_{q-1}$ is surjective for $i=1,2$ and $q \in \mathbf{N}$.

Proof. This is shown by induction on $q$. The case of $q=1$ follows from the fact that $I\left(e_{i}, \mathbf{Z} f_{i}\right)=\mathbf{Z} \quad(i=1,2)$. Assume that $l_{q}: e_{2} \otimes L_{q} \rightarrow L_{q-1}$ is surjective. Then for any $x \in L_{q}$ there exists $y \in L_{q}$ such that $l_{q}\left(f_{1} \otimes x\right)$ $=l_{q}\left(e_{2} \otimes y\right)$. This means that $z:=f_{1} \otimes x-e_{2} \otimes y$ belongs to $L_{q+1}$ by definition. Since $l_{q+1}\left(e_{1} \otimes z\right)=I\left(e_{1}, f_{1}\right) x-I\left(e_{1}, e_{2}\right) y=x$, this implies the surjectivity of $e_{1} \otimes L_{q+1} \rightarrow L_{q}$.

In a symplectic lattice $H$, one can choose $e_{1}=e_{2}=a$ and $f_{1}=f_{2}=b$ for the symplectic pair $a$ and $b$. Thus $l_{n}$ is surjective for $n \geq 0$.

Return to the proof of the surjectivity of $l_{p q}$ for $p>1$. Consider the following natural commutative diagram.

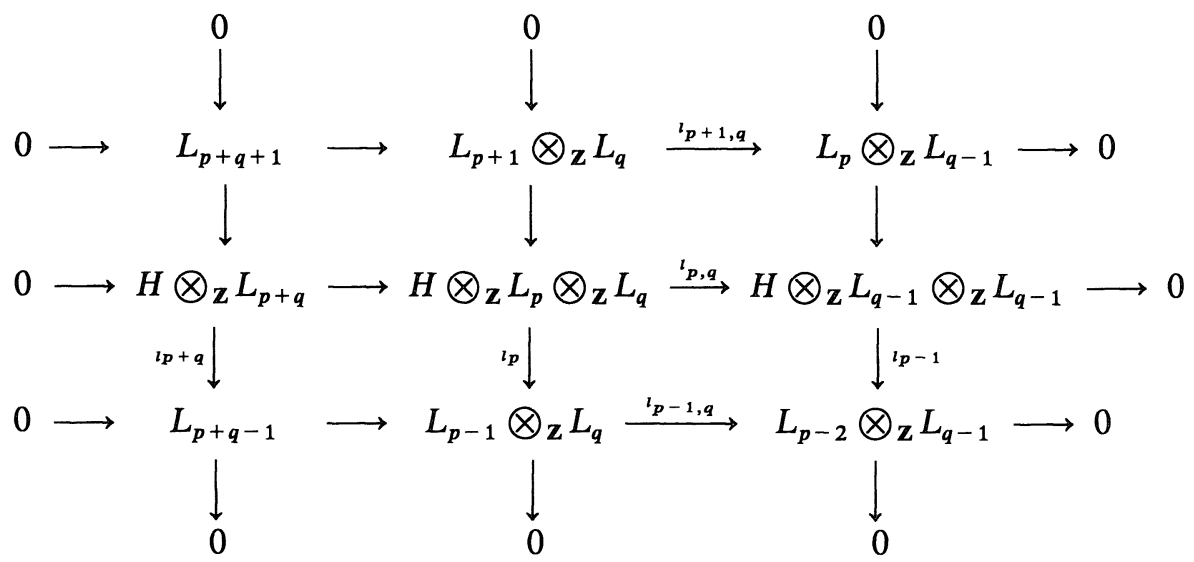


By assumption, all columns are exact. By the induction hypothesis the bottom and the middle rows are exact. Hence the first row is exact. This completes the induction on $p$ and the proof of the Lemma 1 .

2. In view of the description of (A.4), the bilinear form $I^{\otimes n}$ on $H^{\otimes n}$ induces a bilinear form $I_{n}$ on $L_{n}$, which is symmetric or skew symmetric according as $n$ is even or odd. The fact that $I_{n}$ are compatible with the inclusion maps is obvious.

Let us consider the formal power series: $f(t):=\sum_{n=0}^{\infty}\left[L_{n}\right] t^{n}$. Then (A.3) implies: $0 \rightarrow \frac{d}{d t}(t f(t)) \rightarrow f(t)^{2} \rightarrow t^{2} f(t)^{2} \rightarrow 0$, which can be solved to give $f(t)=(1$ $\left.-[H] \cdot t+t^{2}\right)^{-1}$. In particular we have

$$
\sum_{n=0}^{\infty} \operatorname{rank}\left(L_{n}\right) t^{n}=\left(1-2 g \cdot t+t^{2}\right)^{-1}
$$

and hence an explicit formula:

$$
\operatorname{rank}\left(L_{n}\right)=\sum_{k=0}^{[n / 2]}(-1)^{k}\left(\begin{array}{c}
n-k \\
k
\end{array}\right)(2 g)^{n-2 k} .
$$

Remark 1. The construction of the sequence $L_{n}$ can be started from any lattice $H$. The condition of the Assertion is satisfied by a wide class of lattices (e.g. one containing a unimodular sublattice of rank $\geq 2$ ), and the exactness (A.3) holds also for them.

2. Let us denote by $P_{n}(g)$ the polynomial of the right-hand side of the formula for $\operatorname{rank}\left(L_{n}\right)$. Then $P_{n}(g)=0$ has $n$ distinct real roots in $g$, which separates the roots of $P_{n+1}(g)=0$.

Now, we are able to describe graded pieces of a 0 -regular minimal module in terms of integral lattices $L_{n}$.

Lemma. Let the setting be as in (3.4). Assume that $\mathscr{F}$ is 0-regular. Then there is a canonical isomorphism

$$
F_{i+1} / F_{i} \simeq L_{i} \otimes_{\mathrm{z}} \overline{H^{1}\left(\Gamma_{g}, \mathscr{F}\right)}
$$

Proof. This is proved by an induction on $i$, where $i=-1$ is trivial by convention that both sides are zero. The case $i=0$ is proved in Lemma (2.4.2).

The exact sequence (A.2) induces a corresponding exact sequence for $L_{i} \otimes_{\mathrm{z}} \overline{H^{1}\left(\Gamma_{g}, \mathscr{F}\right)}$. A comparison of this exact sequence with that of (3.6.4) implies (A.5). 


\section{References}

[1] Beilinson, A. A., Manin, Y. I. and Schechtman, V. V., Sheaves of the Virasoro and NeveuSchwartz Algebra, Lect. Note in Math., 1289, Springer, 1987.

[2] Bers, L., Spaces of Riemann surfaces, Proc. Int. Congr. Math. Cambridge, (1958), 349-361.

[3] Bol, G., Invarianten linearer Differentialgleichungen, Abh. math. Seminar Hamburger Univ., 16 (1949), 1-28.

[4] Brown, K. S., Cohomology of Groups, Springer-Verlag, 1982.

[5] — Eine Verallgemeinerung der Abelschen Integrale, Math. Z., 67 (1957), 267-298.

[6] Eichler, M., Grenzkreisgruppen und kettenbruchartige Algorithmen, Acta Arithmetica, 11 (1965), 169-180.

[7] Goldman, William M., The Symplectic Nature of Fundamental groups of Surfaces, Adv. in Math., 54 (1984), 200-225.

[8] Hitchin, N. J., The self-duality equation on a Riemann surface, Proc. London Math. Soc., 55 (1987), 59-126.

[9] Hubbard, John H., The monodromy of projective structures, Riemann Surfaces and Related Topics, Ann of Math., (1980) Princeton University Press, 257-275.

[10] Kodaira, K. and Spencer, D. C., Existence of complex structure on a differentiable family of deformations of compact complex manifolds, Annals of Math., 70 (1959), 145-166.

[11] Lyndon, R. C., Cohomology theory of groups with a single defining relation, Ann. of Math., 52 (1950), 650-655.

[12] Murakami, S., Vanishing Theorems on Cohomology Associated to Hermitian Symmetric Spaces, Ann. Inst. Fourier, Grenoble, 37 (1987), 225-233.

[13] Pekonen, O., Contributions to and a survey on Moduli spaces of differential geometric structures, Ber. Math. Inst. Univ. Jyväskylä (Finlande), 1988, Preprint.

[14] Saito, K., On the Moduli Space for Fuchsian Groups, Algebraic Analysis, Academic Press, II, (1988), 735-787.

[15] Shimura, G., Sur les intégrales attachées aux formes automorphes, J. Math. Soc. Japan, 11 (1959), 291-311.

[16] - Arithmetic theory of Automorphic Functions, Iwanami Shoten, Princeton Univ. Press, 1971.

[17] Siegel, C. L., Some remarks on discontinuous groups, Ann. of Math., 46 (1945), 708-718.

[18] Tromba, A. J. and Fischer, A. E., Almost complex principal bundles and complex structure on Teichmüller space, J. reine angew. Math., 352 (1984), 151-160.

[19] Weil, A., Remarks on the cohomology of groups, Ann. of Math., 80 (1964), 149-157.

[20] Wolpert, S., On the symplectic geometry of deformations of a hyperbolic surface, Ann. of Math., 117 (1983), 207-234.

[21] $\longrightarrow$ On the Weil-Petersson Geometry of the Moduli Space of Curves, Amer. J. Math., 107 (1985), 969-997.

[22] Labute, J. P., On the decending central series of groups with a single defining relation, Journal of Algebra, 14 (1970), 16-23.

[23] Kohno, T. and Oda, T., The Lower Central Series of the Pure Braid Group of an Algebraic Curve, Adv. Studies in Pure Math., 12 (1987), 201-219.

[24] Saito, K., Higher Eichler integrals and Vector Bundles over the Moduli of Spinned Riemann surface, Lect. Note in Math., 1468, Springer, 1990. 